\title{
Theoretical Aspects of a Discrete-Binding Approach in Quartz-Crystal Microbalance Acoustic Biosensing
}

\author{
Vasilios Raptis, ${ }^{1}$ Achilleas Tsortos, ${ }^{1, *}$ and Electra Gizeli ${ }^{1,2}$ \\ ${ }^{1}$ Institute of Molecular Biology and Biotechnology, FO.R.T.H, 70013 Heraklion, Greece \\ ${ }^{2}$ Department of Biology, University of Crete, Heraklion 71409, Greece
}

(Received 10 August 2018; revised manuscript received 25 October 2018; published 13 March 2019)

\begin{abstract}
We present an elaboration of the discrete molecule binding theory in acoustic biosensing, as proposed by Tsortos (Biophys. J. 2008). Essentially, the parameters of a damped harmonic oscillator model are mapped to attributes of the sensor crystal and analyte molecules attached on it in the presence of water. This way, changes in wave frequency $\Delta f$ and energy dissipation $\Delta D$ are linked with molecular properties, which can be evaluated by fitting the model to experimental data. The theory attempts to interpret measurements at low to medium levels of surface coverage where the film-formation assumption breaks down. The established linear relationship between the acoustic ratio $\Delta D / \Delta f$ and intrinsic viscosity $[\eta]$, a structural hydrodynamic parameter of the analyte, is recovered. Moreover, possible semi-quantitative explanations are offered for the experimentally observed nonzero intercept and the plateau reached by $\Delta D / \Delta f$ when very short or very long molecular chains are measured. These incorporate various system parameters such as the character of the linker, the shape/size of the molecules, and the hydrodynamic interactions taking place at the sensor's surface in solution. The presented model is formulated to hold for the quartz crystal microbalance (QCM-D) sensor and sheds new light in many standing questions regarding its operation with biological samples in liquid.
\end{abstract}

DOI: 10.1103/PhysRevApplied.11.034031

\section{INTRODUCTION}

Shear-wave acoustic devices work by forcing a piezoelectric material at the core of the device to vibrate and following the changes of the crystal's shear oscillatory motion that take place when biological or other matter is in contact with the moving surface. Theoretical understanding of the factors that affect the signal of the quartz crystal microbalance (QCM-D sensor), the Love-wave sensor, but also other types of acoustic devices, is crucial in studying the properties of a given system and/or designing better detection assays. Thorough understanding of some systems has been established within decades of research. Theoretical developments include the Sauerbrey equation linking the wave frequency shift $(\Delta f)$ with the mass of a purely rigid homogeneous thin film [1], approaches in nanotribology [2,3], and the Gordon-Kanazawa model that accounts for the wave-energy dissipation $(\Delta D)$ processes in the presence of a purely viscous layer in contact with the oscillating crystal surface $[4,5]$. In many experiments where the "purely rigid" or the "purely viscous" cases do not apply, the formation of a film by the attached material is assumed and models have been developed in

\footnotetext{
*atsortos@imbb.forth.gr
}

order to understand such structures having in common the description of viscoelasticity of the film/layer [6-11].

In biology, many systems of interest are in the form of analyte solutions, for example, proteins, DNA, RNA, and so on; when adsorbed at a sensor's surface, film models can be very useful provided their assumptions for a continuous and homogeneous system are more or less satisfied. Under conditions of sparse or unevenly distributed coverage, the film approximation tends to break down. In the extreme case of a single molecule grafted on the surface, there would be some (very weak) signal, but clearly no film formation. Alternative approaches are then required, taking into account the discrete or heterogeneous character of such systems, especially when analysis is performed not in air but in the presence of a solvent that calls for hydrodynamics to be considered.

The problem of nonuniformly deposited layers [12-14] or discrete systems in acoustic sensing has not gone unnoticed by the acoustic sensing community. A direct approach is to explicitly consider the case of single particles attached to the sensor surface via a specific linker. Generally, the questions addressed concern the effect of the linker, particle-fluid coupling, particle-particle interactions, and the associated problem of the role of surface coverage, as well as the role of size, shape, and internal degrees of freedom of these (analyte) particles. In 
one case, experimental frequency shifts of quartz crystals with deposited gold particles have been interpreted using a model of two coupled oscillators to account for crystal elasticity and the surface-particle adhesive bond [15]. An extension of that model [16] added quartz and particle damping, dry friction as well as nonlinear-adhesive bonding. In agreement with actual observations, the model predicted that when the surface-particle bond is weaker than a well-defined threshold, it leads to increasing frequency with added particles, whereas a stronger link predicts a negative frequency shift, as is commonly measured. When the particle-surface bond is relatively flexible, some energy is dissipated in the contact zone. Twodimensional finite-element calculations emulating rigid (mathematical) spheres at various surface coverages have been carried out to analyze the QCM signal as a function of linker compliance, particle size, and surface coverage $[17,18]$. Combined with experiments, it was shown that dissipation increased for narrow and flexible linkers; the ratio of resonance width $\Delta \Gamma$ (or $\Delta D$ ) over negative frequency shift $(\Delta \Gamma / \Delta f)$ tended to decrease with surface coverage. Energy dissipation exhibited a maximum at moderate coverage, attributed to the freedom of particles to rock and rotate and interact hydrodynamically with their neighbors; increasing coverage restricts freedom of mutual reorientation, thus reducing dissipation. Extrapolating the ratio $\Delta \Gamma / \Delta f$ to zero dissipation yields a cut-off frequency that was interpreted as a "Sauerbrey limit," allowing to determine the particle size from the film thickness [18].

Lattice-Boltzmann (LB) simulations showed that the geometry of adsorbate depositions can affect the acoustic signal because of the different amounts and mechanisms of solvent entrapment [19]. In order to separate the effect of surface coverage from fluid-particle coupling, LB simulations of an isolated spherical particle have also been combined with dimensional analysis to derive an expression for the QCM frequency shift of rigidly attached spherical particles [20]. In it, three terms are summed, namely a "Sauerbrey" contribution, a term related to displaced fluid mass, and a viscous term proportional to penetration depth; the latter introduces a dependence of frequency shift, $\Delta f / n$, on the overtone, $n$, even for rigid attachment. The model assumes low particle deformation and low surface concentration so that particle-particle hydrodynamic interactions can be neglected. Under such conditions, particle size as well as surface coverage can be reasonably estimated. The method was extended to correlate the aspect ratio of ellipsoid particles at vanishing surface coverage, with the slope and intercept of a penetration depth vs $\Delta f / n$ plot. Finally, the same approach was used to look at the effect of surface coverage and interpret the decrease in frequency and dissipation shift beyond a cut-off coverage as the result of hydrodynamic interactions. The data provided a theoretical basis for determining particle sizes from QCM readings at the limit of full coverage [20,21].

Whereas some of these works provide a way to determine the size of noncompliant analyte particles with low aspect ratio, they all remain without any clear reference to particular analyte molecule characteristics. Tsortos et al. [22-24] followed a route that allowed to look at the role of the actual size and shape of rodlike molecules, such as DNA linear chains, or even more complex structures using nucleotides as building blocks. In particular, they relied on solution viscosity theory to show that the ratio of change in wave-energy dissipation $\Delta D$, over frequency shift, $-\Delta f$, (termed "acoustic ratio," $\Delta D / \Delta f$ ), is independent of surface coverage and proportional to the analyte's intrinsic viscosity $[\eta]$, a hydrodynamic quantity directly related to structure [23]. An equivalent result was also derived for the ratio of wave amplitude change over phase shift, $\Delta A / \Delta \mathrm{Ph}$, measured by Love-wave acoustic devices [22]. Experiments showed the relation to hold for DNA regardless of surface coverage $[22,23,25,26]$, whereas extrapolation to zero coverage was required for a number of different protein configurations [27] in order to find a similar dependence on $[\eta]$. Some open questions of particular interest in this approach include the experimental findings of nonzero DNA acoustic ratio at the zero $[\eta]$ limit and linearity break-down (reaching a plateau) beyond a certain cut-off value located at approximately 500-600 base pairs for double-stranded DNA [24].

Intrinsic viscosity is intimately related to structure, namely, molecular weight and other size and shape indicators; slender particles have greater $[\eta]$ than objects with a low aspect ratio. Quantitative correlations of these factors can be obtained either experimentally (viscometry) $[24,28-30]$ or theoretically [30-33] and computationally $[29,34-36]$. Because of the link with the acoustic ratio, the theory by Tsortos allows to study whole families or homologous series of species of various sizes, shapes, and conformations in a systematic manner. As it is based on solution concepts, the theory is suitable for sparsely anchored molecules that do not necessarily form continuous films, but make up a collection of discrete particles bound at the crystal surface. The theory holds provided the molecules are free-dangling and hydrodynamically independent in the sense that shape is not affected by anchoring or by lateral crosstalk. This approach, so far, has been exploited in a variety of projects: to obtain information on the length of surface-hybridized DNA for signal amplification [37], on the elongation of peptide fibrils [38], to study the reversible transitions of a DNA proton-driven nanopump [39] and the orientation of peptides [40], the conformation of aptamers [41] and ssDNA [42] at surfaces and, of course, in the analysis of viscoelasticity of bio/organic surface films [18,43-46]. In our laboratory, its usefulness in biotechnology and biophysics has been explored by detecting DNA hybridization [47-50], 
DNA/protein interactions [51], and protein [25,27] and DNA structural characterization [25,26,52,53]; we also suggested experimental methods to use [25] and tested the generality of the theory [24].

In this work, we elaborate on the theoretical foundations of the above approach [22] by combining the mechanics $[15,54]$ of damped harmonic oscillators with hydrodynamic considerations and we derive explicit expressions for QCM-D energy dissipation and frequency change as functions of the analyte properties. The model assumes analyte molecules that are attached on the surface, but retain a certain orientational freedom, depending on their internal flexibility as well as the stiffness of particlesurface link; examples of such species include biotinylated DNA attached on neutravidin, proteins, polymers, and so on. Following a number of assumptions and appropriate algebraic manipulations, we map the system properties to the dissipation factor and natural frequency of a onedimensional damped harmonic oscillator. We combine, in a simple manner, inertial terms and the crystal's internal friction with surface-liquid viscous coupling, but also dissipative contributions due to the presence of attached particles. The model (a) extends the previous discretebinding approach [22], which is recovered as a limiting case, (b) predicts a number of additional features present in actual measurements, linking them directly to parameters related to analyte and overall system properties, and (c) provides possible answers to two open questions, that is, the existence of an experimentally observed "plateau" and a nonzero $Y$ intercept in the $\Delta \mathrm{D} / \Delta f$ vs DNA length plots [22-24].

We start in Sec. II, where our theoretical assumptions are presented and the model's fundamental expressions are derived. In Sec. III, the model is validated by comparing predictions based on reasonable assumptions with experimental acoustic measurements of DNA (QCM-D, $35 \mathrm{MHz}$ ). Results and further possibilities as well as limitations and open questions are discussed in Sec. IV. In Sec. V, our conclusions are summarized and future directions are briefly outlined. In Appendix A, expressions are presented for the frequency in air, in contact with pure solvent, and in the presence of analyte; also, the solution of the set of equations comprising the model is discussed. Finally, in Appendix B, an approximate expression is derived at the limit of vanishing analyte mass per unit surface area, leading to the original theory [22-24].

\section{THE MODEL}

\section{A. Setup}

When an appropriate voltage is applied to the crystal of a QCM device, mechanical deformation in the form of standing waves will develop across the piezoelectric material. The sample to measure is deposited on the surface, therefore, users are interested in the normal modes of surface oscillations. This condition is satisfied by the odd harmonics that have the appropriate wavelength; experiments referred to in this work are conducted using the seventh harmonic of QCM-D, namely, $35 \mathrm{MHz}$. Our theoretical approach treats the crystal surface as a one-dimensional damped harmonic oscillator endowed with its own inertia, internal friction, and elasticity. These are merely effective parameters, such that the oscillator's natural frequency and dissipation in pure solvent coincide with the experimentally observed ones; they have nothing to do with the actual crystal properties. The only property pertinent to the actual crystal is the surface area.

We work with an idealized setup depicted in Fig. 1. The surface is rigid, perfectly flat, and does not tilt or rotate, but only executes in-plane oscillations along the $X$ axis; the $Z$ axis, which will not play any role in our analysis, also lies on the surface. A particle of analyte material, depicted here as a sphere (but generally of an arbitrary shape) is attached to the surface through a bond (linker) shown as a straight line. The exact distance of the particle from the surface does not play a major role in the subsequent derivations; generally, it can be identified with the position of the particle's center of mass. For biomolecular and other chain molecules, it can be assumed to lie between the radius of gyration, $R_{g}$, and half the contour length, $L_{c}$, of the molecule. For dsDNA (the analyte of interest here) with a given number of base pairs (bp), the radius of gyration is given as $R_{g}(\mathrm{~nm})=0.162(\mathrm{bp})^{0.878}$ for chains with $\lesssim 2000 \mathrm{bp}$ and as $R_{g}(\mathrm{~nm})=2.08(\mathrm{bp})^{0.515}$ for longer ones [29] and the contour length is readily found as $L_{c}(\mathrm{~nm})=0.34(\mathrm{bp})$.

The importance of bonds (number of them, strength, and so on) and their effect on the measured frequency and energy dissipation has been the focus of many studies $[15$, 17,55-58]. In this work, the bond is allowed to change orientation under the influence of internal and external forces. Although combinations of elastic springs or even more sophisticated potentials can be used to emulate restoring forces, here, a length-preserving tension along the bond is considered. Admittedly, this is an important concession in favor of tractability; it will be shown though, that predictions based on this setup compare very well with actual measurements. The surface is in contact with the "solvent" and this fluid surrounds the particle. The area of the surface is assumed to be large enough that end effects can be safely ignored. During measurements, $N$ identical analyte particles (not shown in the figure) are also immersed in the solvent and are bound at various positions on the surface. The solvent is a homogeneous fluid with its own density $\rho_{0}$ and viscosity $\eta_{0}$. When the surface oscillates, a shear wave develops through the solvent in the form of a horizontal velocity profile that decays exponentially with distance from the surface along the $Y$ axis (Fig. 1).

As an operating protocol, we consider what actually happens during a typical sensing acoustic experiment with 


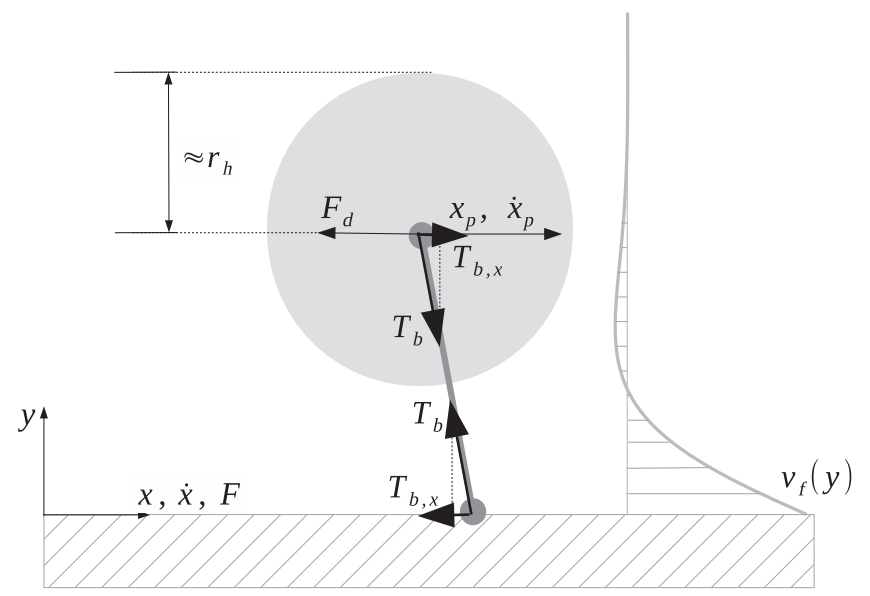

FIG. 1. An idealized illustration of a QCM-D device operating in liquid with analyte molecules on the crystal surface. An analyte particle depicted as a sphere is attached via a bond (thick gray line) on the crystal (hatched region). The external driving force $F$ is exerted along the $x$ axis, which is also the direction of motion of the surface, the particle, and the surrounding fluid. The latter exhibits a velocity profile $v_{f}$ (not in scale) that decays exponentially with distance $y$ from the surface. The particle position is denoted by $x_{p}$. A tension force $T_{b}$ is exerted along the bond. Its horizontal component, $T_{b, x}$, is exerted on the particle and the surface (in opposite directions). The particle is also subjected to a drag force $F_{d}$ that depends on the particle's velocity relative to that of the surrounding fluid and its apparent hydrodynamic radius, $R_{h}$.

frequency and energy dissipation monitoring. At the beginning, the sensor is operating in pure solvent. An external driving force that is intermittently switched on and off varies sinusoidally at an appropriate driving frequency $f_{d}$, usually very close to the system's resonance frequency in order to maximize amplitude. When the force is on, a short transient period of oscillations of increasing amplitude is followed by a steady-state period; it is assumed that the time the force is on suffices to establish steady-state oscillations. Then the force is turned off, surface oscillations die off exponentially, and the so-called ring-down frequency and decay rate are recorded. The energy dissipates due to both the crystal's internal friction and the solvent's resistance to shear. To measure the analyte of interest, a solution will pass through the chamber until $N$ molecules attach randomly to binding sites on the surface. Then, pure solvent follows and any excess material is washed away leaving only tethered molecules behind. Meanwhile, the sensor will keep oscillating and relaxing alternately, as is customarily done in "ring-down" QCM-D experiments.

\section{B. Equations of motion}

The "crystal + analyte" system will be characterized by certain changes with respect to the unloaded sensor, namely, an increase in mass, the presence of drag forces exerted on the analyte molecules, and a change in viscosity of a fluid layer in contact with the sensor from $\eta_{0}$ to $\eta$. We are going to correlate these changes with experimentally measured quantities, that is, the energy dissipation factor $D$ and the frequency $f$ or its angular equivalent $\omega$ (of note, frequencies in our analysis are not divided by the overtone number). We begin by writing down the equation of motion of the oscillating surface for the following three cases: vacuum, pure solvent, and analyte addition. When operating in vacuum (or in the air, assuming the air's resistance is negligible) the oscillator is characterized by an effective inertia, internal friction, and elasticity

$$
\underbrace{m_{s} \ddot{x}}_{\begin{array}{c}
\text { effective } \\
\text { inertia }
\end{array}}+\underbrace{\kappa \dot{x}}_{\begin{array}{c}
\text { internal } \\
\text { friction }
\end{array}}+\underbrace{K x}_{\begin{array}{c}
\text { internal } \\
\text { elasticity }
\end{array}}=F(t),
$$

where $x$ is the surface's displacement and overdots denote time derivatives; $m_{s}$ is the unloaded crystal's effective mass, $\kappa$ is a friction coefficient, $K$ is an elasticity constant, and $F(t)$ is the external driving force.

When operating in pure solvent, a term due to the fluid's resistance to shear is added (the opposite force is exerted on the fluid by the surface). The equation of motion becomes

$$
m_{s} \ddot{x}-\underbrace{\left.\eta_{0} A \frac{\partial v_{f, 0}}{\partial y}\right|_{y=0}}_{\text {resistance to shear }}+\kappa \dot{x}+K x=F(t) .
$$

Here, $\eta_{0}$ is the viscosity of the overlaying fluid, $A$ is the crystal's surface area, $v_{f, 0}$ is the fluid's velocity, which is generally a function of time and position, and $y$ is the distance from the surface. Generally, the stress term in Eq. (2) should be an average over the crystal surface. Here, it is assumed that the velocity profile $v_{f, 0}$ is independent of $x$ or $z$, which is reasonable given the problem setup, so using the particular form of the viscous resistance term is legitimate. In fact, the steady-state regime corresponds to the well-known Stokes' second problem [59] and its solution will be used in our analysis.

Finally, when analyte is attached on the surface, we write two equations of motion: one for the surface and one for the analyte molecule. Due to the presence of the particle-surface bond, a tension force $T_{b}$ exists. The horizontal component $T_{b, x}$ of this force is added to the two equations of motion. We consider a number of $N$ molecules of hydrodynamic (Stokes) radius $R_{\mathrm{h}}$ attached on the surface as shown in Fig. 1. The movement of these molecules along the $X$ direction is guided by the horizontal component $T_{b, x}$ of the tensions along their surface-particle bonds. An equal tension force of opposite direction is transferred to the attachment point on the surface. Then, 
the equation of motion for a loaded sensor is

$$
m_{s} \ddot{x}-\left.\eta A \frac{\partial v_{f, 0}}{\partial y}\right|_{y=0}+\underbrace{\sum_{i=1}^{N} T_{b, x, i}}_{\text {bond tensions }}+\kappa \dot{x}+K x=F(t),
$$

where the solvent viscosity, $\eta_{0}$, has been replaced by a new viscosity, $\eta$, in the shear force term. In what follows, 0 subscripted symbols will denote quantities referring to operation in pure solvent while $s$ subscripts refer to intrinsic properties of the quartz crystal and $p$ subscripts to the analyte particle. We now write the equation of motion for each particle of mass $m_{p}$ :

$$
m_{p} \ddot{x}_{p}+\underbrace{6 \pi \eta R_{h}\left(\dot{x}_{p}-v_{f}\right)}_{\text {drag }}=T_{b, x},
$$

where $x_{p}$ is the particle's displacement parallel to the crystal surface and $v_{f}$ is the velocity of fluid surrounding the particle. A Stokes-like drag term is written in terms of analyte molecule velocity relative to the fluid and its apparent hydrodynamic radius $R_{h}$; the unknown tension $T_{b, x}$ acts as an external force driving the particle. The same viscosity $\eta$ as in Eq. (3) is used in the context of a "mean-field" approximation in order to incorporate the effect of all hydrodynamic and other kinds of interactions in a few parameters. In other words, each analyte molecule feels the effect of a solution with properties determined by the presence of all other molecules and the crystal surface.

Due to the nonsteady (accelerating) motion, added-mass effects are present [60]. These are more complicated than just an extra inertial term because of the nearby surface. However, we can roughly approximate their contribution by the mass of fluid displaced by the particle times the particle's acceleration relative to the fluid (i.e., treating the chains as cylinders). Based on arguments that we present in following paragraphs, this contribution can be shown to be very small: $N m^{\prime}\left(\ddot{x}_{p}-\dot{v}_{f}\right)<<m \ddot{x}$ where $m$ is the total oscillator inertia and $m^{\prime}$ is the added mass. To avoid very complicated expressions, we adopt the assumption of negligible added mass from the very beginning and describe the particle dynamics by means of Eq. (3a). Nonsteady motion also implies that retarded viscous contributions summing up to the so-called Basset-Boussinesq force [61] should be accounted for. However, the oscillatory nature of the motion leads to partial cancellation of these contributions; the small remainder is approximately proportional to the current particle velocity relative to the surrounding fluid and we chose to take it as part of the effective Stokes term.

The fluid velocity profile has also changed, but it will be assumed to have the same general form as in the absence of analyte; in the steady-state regime, this form is a wellknown solution for a fluid in contact with an in-plane oscillating surface. Assuming that the surface steady-state velocity can be written as $\omega x_{\max } \cos \omega t$ and that the no-slip condition holds [45], this solution is given by the expression

$$
v_{f}(y, t)=v_{f, y=0} e^{-k y} \cos (\omega t-k y),
$$

[59] where the wavenumber $k$ is a function of fluid viscosity $\eta$, density $\rho$, and oscillation angular frequency $\omega$, namely,

$$
k=\sqrt{\omega \rho / 2 \eta}
$$

The inverse quantity, $\delta=k^{-1}$, defines the shear-wavedecay length also known as the penetration depth. It should be noted that $\omega$ is the system's natural angular frequency that generally differs from $\omega_{d}$, the driving force oscillation frequency. For operation in pure solvent, 0 subscripted quantities should be used in Eqs. (4) and (4a). To be able to do that, that is, use the same equation in solvent and analyte solution, we postulate that disturbance of the fluid's flow by the introduction of new analyte molecules is minimal; in particular, apart from small changes in $k$ and $\omega$, the fluid's motion away from the analyte molecules is not greatly perturbed by their presence. On the other hand, the motion of the analyte molecules is nontrivially affected by the fluid through the effective drag force, Eq. (3a). Normally, the assumption of a minimally disturbed fluid requires sparse coverage; the extent to which it remains valid with denser systems will be discussed in detail in subsequent paragraphs.

Equation (4) serves as the first step toward the formulation of our damped harmonic-oscillator model; starting from that, it is easily shown that

$$
\left.\frac{\partial v_{f}}{\partial y}\right|_{y=0}=-k\left(\dot{x}+\frac{\ddot{x}}{\omega}\right),
$$

or an analogous 0 subscripted expression for operation in pure solvent. If we apply the above result to Eq. (2), we find

$$
\underbrace{\left(m_{s}+\eta_{0} \frac{A k_{0}}{\omega_{0}}\right)}_{m_{0}} \ddot{x}+\left(\eta_{0} A k_{0}+\kappa\right) \dot{x}+K x=F(t),
$$

where we have also defined the effective inertia of the crystal, $m_{0}$, operating in contact with pure solvent. Equation (6) resembles an equation of motion of a linear dampedharmonic oscillator subjected to an external driving force. The additional term $\eta_{0} A k_{0} / \omega_{0}$ in the acceleration coefficient is a kind of "added mass" (not to be confused with 
the effects of unsteady motion discussed earlier) that represents the inertia of liquid entrained by the moving surface. In a similar vein, if we combine Eq. (3) with Eq. (5), we obtain

$$
\left(m_{s}+\eta \frac{A k}{\omega}\right) \ddot{x}+(\eta A k+\kappa) \dot{x}+\sum_{i=1}^{N} T_{b, x, i}+K x=F(t) .
$$

This equation is analogous to Eq. (6) except for an extra term summing the bond tensions.

The next step is to eliminate this term in order to bring Eq. (7) to the final form of a damped harmonic oscillator; this can be done using Eq. (3a), which is written in terms of the molecule's displacement, $x_{p}$. Because of the bond with the surface, we expect a certain coordination of the molecule's movement with surface oscillations. We try to find an explicit expression for this coordination effect. If we rearrange Eq. (3a) as

$$
\ddot{x}_{p}=-\frac{6 \pi \eta R_{h}}{m_{p}}\left(\dot{x}_{p}-v_{f}\right)+\frac{T_{b, x}}{m_{p}}=-\frac{1}{\tau_{p}}\left(\dot{x}_{p}-v_{f}\right)+\frac{T_{b, x}}{m_{p}},
$$

we note that the particle's velocity relative to the fluid $\left(\dot{x}_{p}-v_{f}\right)$ acts as a driving force that tends to equate the analyte molecule velocity to that of the fluid; the parameter $\tau_{p}$ is defined as

$$
\tau_{p}=\frac{m_{p}}{6 \pi \eta R_{h}},
$$

which represents a time scale related to this process.

We may consider now as an example double-strand DNA in biosensing. Its hydrodynamic radius is given as $R_{h}(\mathrm{~nm})=0.3145(\mathrm{bp})^{0.652}$ for chains with $\lesssim 2000 \mathrm{bp}$ and as $R_{h}(\mathrm{~nm})=0.795(\mathrm{bp})^{0.573}$ for longer ones [29]. For a $100 \mathrm{kDa}$ molecule (i.e., approximately $150 \mathrm{bp}$ ), $R_{h}$ is calculated to be $8.3 \mathrm{~nm}$ and then $\tau_{p}$ is approximately equal to $0.53 \mathrm{ps}$, a very short time in comparison with the time scales of sensor oscillations (e.g., five-orders of magnitude shorter than the period of $35 \mathrm{MHz}$ oscillations). This result, which is also suggestive of our minimal-fluiddisturbance assumption, implies a competition between bond tension and drag by the fluid, resulting in a phase lag, $\varphi$, of the particle's motion with respect to the surface's oscillation. Then, the particle's velocity and acceleration will be

$$
\begin{aligned}
& \dot{x}_{p}=v_{p, \max } \cos (\omega t+\phi) \quad \text { and } \\
& \ddot{x}_{p}=-\omega v_{p, \max } \sin (\omega t+\phi),
\end{aligned}
$$

where $v_{p, \max }$ is the amplitude of the particle velocity. We can realistically assume that $\varphi$ varies with distance from the surface or particle size and generally ranges between zero (near the surface) and $-k y$ (away from the surface, provided that the linker and analyte molecule are not totally rigid). We also expect that $v_{p, \max }$ should vary with particle distance or size, ranging between the surface velocity amplitude (very near the surface) and zero (far away). Then, a short chain corresponding to a particle very near the surface, as well as the fluid at that distance, should have very small phase lags, $\varphi \approx-k y \approx 0$. Correspondingly, the oscillation amplitude of the particle and fluid alike should be comparable with each other and somewhat smaller than the surface's oscillation amplitude, so we can write $\dot{v}_{p} \propto \ddot{x}$. Long chains modeled as particles situated far away from the surface can be largely out of phase with respect to the surface. However, the amplitude of their oscillations will be small and so will the error associated with the in-phase assumption for these species.

If we combine Eqs. (3a), (7), and the above assumption about in-phase oscillations, we can find

$$
\begin{gathered}
\underbrace{\left(m_{s}+\eta \frac{A k}{\omega}+m_{p} \sum_{i=1}^{N} a_{i}\right)}_{m} \ddot{x}+(\eta A k+\kappa) \dot{x} \\
+6 \pi \eta R_{h} \sum_{i=1}^{N}\left(\dot{x}_{p}-v_{f}\right)+K x=F(t),
\end{gathered}
$$

where $a_{i}$ are unknown proportionality coefficients and we can also define the effective inertia of the crystal in liquid with adsorbed analyte as $m$.

To bring Eq. (10) to a form analogous to Eq. (6), we need to eliminate the fluid velocity. To this end, we define a new quantity, the equivalent hydrodynamic radius $r_{h}^{\mathrm{eq}}$. This is the hydrodynamic radius of a particle that is otherwise identical to an analyte molecule, moves through a quiescent fluid with the same properties $(\eta, \rho)$ as our system in perfect coordination with the oscillating surface, and suffers a drag force that does the same work during an oscillation period, $T$, as the drag exerted on the actual analyte particles. The last condition can be expressed as

$$
\begin{aligned}
& r_{h}^{\mathrm{eq}} \int_{x(0)}^{x(T)} \dot{x} d x=R_{h} \int_{x_{p}(0)}^{x_{p}(T)}\left(\dot{x}_{p}-v_{f}\right) d x_{p} \quad \text { or } \\
& r_{h}^{\mathrm{eq}} \int_{0}^{T} \dot{x}^{2} d t=R_{h} \int_{0}^{T}\left(\dot{x}_{p}-v_{f}\right) \dot{x}_{p} d t .
\end{aligned}
$$

The equivalent radius $r_{h}^{\text {eq }}$ will be smaller (possibly by orders of magnitude) than the actual hydrodynamic (Stokes) radius $R_{h}$ because the integrands in Eq. (11) will also be small according to our preceding discussion on phase lag $\varphi$. Later, it will be shown that $r_{h}^{\text {eq }}$ can 
be correlated with experimental evidence from acoustic measurements.

Using Eq. (11), the equation of motion, Eq. (10), is simplified to

$$
\begin{aligned}
& \left(m_{s}+\eta \frac{A k}{\omega}+m_{p} \sum_{i=1}^{N} a_{i}\right) \ddot{x}+\left(\eta A k+\kappa+6 \pi \eta N r_{h}^{\mathrm{eq}}\right) \dot{x} \\
& \quad+K x=F(t) .
\end{aligned}
$$

The coefficients $a_{i}$ should be $<1$, as per our above assumptions about phase lag $\varphi$. The inertial term, pertaining to the entrained fluid, should be larger than its counterpart in Eq. (7) because of the increase in viscosity (as the wavenumber, $k$, decreases with the square root of viscosity and the frequency and density can be taken constant to a first approximation). It is reasonable to assume that the two changes partly cancel out. In fact, using the approximate expression $m \approx m_{s}+\eta_{0} k_{0} A / \omega_{0}+N m_{p}$ in our calculations presented in Sec. III suffices to reproduce experimental results with remarkable accuracy while providing reasonable estimates of analyte and system properties. It should be noted that this approximation concerns only the system inertia as a whole and does not imply the same oscillation amplitudes for surface and particle: apart from taking $a=1$, the entrained fluid inertia is also replaced by its pure solvent counterpart; these two changes at least partially cancel out. Thus, the following form is henceforth adopted for the equation of motion of the analyte-loaded sensor:

$$
\begin{aligned}
& \underbrace{\left(m_{s}+\frac{\eta_{0} k_{0} A}{\omega_{0}}+N m_{p}\right)}_{\approx m} \ddot{x}+\left(\eta A k+\kappa+6 \pi \eta N r_{h}^{\mathrm{eq}}\right) \dot{x} \\
& \quad+K x=F(t) .
\end{aligned}
$$

Making the above assumption about the inertia term is an important step because it implies linear dependence of that term on analyte mass (via the $N \times m_{p}$ product). Generally, on the one hand, we would expect the frequency shift per added analyte molecule to depend on the number of particles already attached on the surface (thus, implying nonlinearity) because of hydrodynamic interactions among particles. On the other hand, mutual cancellation of lateral forces is possible to the extent that the surface is more or less homogeneously covered. Such a scenario may extend the validity of our previous assumption that the fluid's velocity profile [Eq. (4)] will not be perturbed by the addition of analyte except in the sense that the oscillation frequency and wavenumber will both change slightly. It is interesting to check the extent to which these assumptions remain valid by means of a thought experiment. We start by considering a highly dilute system where the attached molecules are situated very far apart from each other. In it, minimal fluid disturbance by the particles can be assumed as discussed in previous paragraphs; then, provided the surface-particle link is not extremely stiff (orientationwise), analyte molecules will have their motion adjusted to the fluid's motion within very short time scales (of the order of ps) as per Eq. (8a) and the related arguments. Now, suppose that one more molecule is attached on the surface. Then viscosity, oscillation frequency, and density will change very slightly leading to a very small change in the fluid's velocity profile wavenumber $k$. This will cause small disturbances to the motion of other analyte molecules that will be damped within the same time scale [Eq. (8a)]. The new analyte molecule will also adjust itself to the movement of the surrounding fluid, and so a concerted wavelike motion of the tethered molecules following the fluid's motion will reemerge very soon, albeit with a slightly altered frequency and wavenumber $k$. The same procedure can be repeated multiple times, adding one particle at a time. Provided that the particle addition rate does not overlap with the inverse time scale, $\tau_{p}^{-1}$ related to the drag force [Eq. (8a)], it will be possible for the system to retain its characteristics, namely an oscillatory motion of the fluid, as in Eq. (4), and a wavelike pattern of the molecules' motion that will tend to adapt to the fluid's velocity profile, even at moderate surface coverage.

It is worth checking the validity of the above scenario with the aid of a simple calculation. Assuming a typical crystal diameter of $14 \mathrm{~mm}$ and an area per binding site of $50 \mathrm{~nm}^{2}$ (see arguments in next Sections), we have about $3 \times 10^{12}$ binding sites. In experiments, it typically takes about 1 min of solution inflow until saturation, so we have just one binding event every about $20 \mathrm{ps}$ over the whole crystal area, on average. This time suffices for disturbances to decay. Furthermore, the next binding events would probably take place far away, so newly introduced molecules would not "feel" each other's presence and disturbances to the fluid would fade out before propagating far enough to overlap. Also, the duration of steady-state oscillations is many orders of magnitude higher than the time it would take for disturbances due to new particles to wither away. Indeed, using known data about the QCM-D device, it is found that the steady-state period is of the order of $10^{8}$ to $10^{9} \mathrm{ps}$.

We close this discussion by noting that thermal fluctuations can contribute to the reorientation and relaxation processes, but such effects are not considered here. All the above assumptions are to be tested by comparing predictions with experiments (see Sec. III).

\section{Derivation}

So far, we have used a number of assumptions and approximations to cast the equation of motion of the acoustic device's quartz crystal in three different operating conditions (in vacuum, pure solvent, solvent and adsorbed 
analyte) in a form akin to a linearly damped harmonic oscillator. Our results are summarized below:

$$
\begin{array}{ll}
\text { Vacuum: } & m_{s} \ddot{x}+\kappa \dot{x}+K x=F(t) \\
\text { Solvent: } & m_{0} \ddot{x}+\left(\eta_{0} A k_{0}+\kappa\right) \dot{x}+K x=F(t) \\
\text { Loaded: } & m \ddot{x}+\left(\eta A k+\kappa+6 \pi \eta N r_{h}^{\mathrm{eq}}\right) \dot{x}+K x=F(t),
\end{array}
$$

where:

$$
m_{0} \equiv m_{s}+\eta_{0} \frac{A k_{0}}{\omega_{0}} \quad \text { and } \quad m \approx m_{s}+\frac{\eta_{0} k_{0} A}{\omega_{0}}+N m_{p} .
$$

Equation (14) can be directly mapped to a well-known theoretical expression, which contains the system's natural frequency and energy dissipation factor. We use the following generic expression

$$
m \ddot{x}+b \dot{x}+K x=F(t),
$$

to outline the procedure. Briefly, an inverse decay time scale is defined as $1 / \tau=b / m$, the angular frequency can be computed as $\omega^{2}=K / m$, and the energy dissipation factor $D$, or inverse quality factor $1 / Q$, is defined as $D \equiv Q^{-1}=1 / \omega \tau$ [62]. With reference to Eq. (14), we define similar 0 subscripted and s subscripted quantities as before. Then, we divide by the effective inertia in each case to obtain the so-called standard form of the equations of motion:

$$
\begin{array}{ll}
\text { Vacuum: } & \ddot{x}+D_{s} \omega_{s} \dot{x}+\omega_{s}^{2} x=\frac{F(t)}{m_{s}} \\
\text { Solvent: } & \ddot{x}+D_{0} \omega_{0} \dot{x}+\omega_{0}^{2} x=\frac{F(t)}{m_{0}} \\
\text { Loaded: } & \ddot{x}+D \omega \dot{x}+\omega^{2} x=\frac{F(t)}{m} .
\end{array}
$$

$D_{s}, D_{0}, \omega_{s}$, and $\omega_{0}$ are readily measurable in QCM-D experiments and are assumed to be known in subsequent derivations. Also, a theoretical expression linking $\omega_{s}, \omega_{0}$, and $\omega$ is shown in Appendix A.

By comparing the elasticity terms in Eqs. (14) and (15), we readily find

$$
\frac{m}{m_{0}} \frac{\omega^{2}}{\omega_{0}^{2}}=1
$$

In the same vein, by equating the coefficients of the drag terms in the two sets of equations, we find

$$
\begin{aligned}
D_{s} \omega_{s} & =\frac{\kappa}{m_{s}}, \quad D_{0} \omega_{0}=\frac{\eta_{0} A k_{0}+\kappa}{m_{0}}, \quad \text { and } \\
D \omega & =\frac{\eta A k+6 \pi \eta N r_{h}^{\mathrm{eq}}+\kappa}{m},
\end{aligned}
$$

which can be combined to give

$$
\begin{aligned}
D_{0} \omega_{0} & =\frac{\eta_{0} A k_{0}}{m_{0}}+D_{s} \omega_{s} \frac{m_{s}}{m_{0}} \text { and } \\
D \omega & =\frac{\eta A k+6 \pi \eta N r_{h}^{\mathrm{eq}}}{m}+D_{s} \omega_{s} \frac{m_{s}}{m} .
\end{aligned}
$$

Then with

$$
\frac{D \omega-D_{s} \omega_{s} \frac{m_{s}}{m}}{D_{0} \omega_{0}-D_{s} \omega_{s} \frac{m_{s}}{m_{0}}}=\frac{\eta}{\eta_{0}}\left(\frac{k}{k_{0}}+\frac{6 \pi N r_{h}^{\mathrm{eq}}}{A k_{0}}\right) \frac{m_{0}}{m},
$$

and using Eq. (16), we find

$$
\begin{aligned}
\frac{D}{D_{0}}= & {\left[\frac{D_{s} / D_{0}}{\omega_{s} / \omega_{0}}+\left(1-\frac{D_{s} / D_{0}}{\omega_{s} / \omega_{0}}\right) \frac{\eta}{\eta_{0}}\left(\frac{k}{k_{0}}+\frac{6 \pi r_{h}^{\mathrm{eq}}}{(A / N) k_{0}}\right)\right] } \\
& \times \frac{\omega}{\omega_{0}} .
\end{aligned}
$$

We have written Eq. (19) as a function of surface area per analyte molecule, $A / N$, because this is a convenient quantity independent of the (generally unknown) number of attached molecules, $N$, and will be used throughout our calculations. Equations (16) and (19) together with the approximation involved in Eq. (14a) form the basis of our model.

Finally, an interesting relation that connects the effective inertia and internal friction coefficient with a crystal's surface area can be derived in the following manner from Eq. (17):

$$
m_{s}=\frac{\kappa}{D_{s} \omega_{s}}=\underbrace{\frac{\kappa+\eta_{0} A k_{0}}{D_{0} \omega_{0}}}_{\text {crystal + fluid }}-\underbrace{\frac{\eta_{0} A k_{0}}{\omega_{0}}}_{\text {entrained fluid, Eq. (6) }}
$$

where

$$
\kappa_{s}=D_{s} \omega_{s}\left(\frac{1-D_{0}}{D_{0} \omega_{0}-D_{s} \omega_{s}} \eta_{0} k_{0}\right) A
$$

and

$$
m_{s}=\left(\frac{1-D_{0}}{D_{0} \omega_{0}-D_{s} \omega_{s}} \eta_{0} k_{0}\right) A \equiv \sigma_{s} A
$$

The coefficient $\sigma_{f}$ depends on quantities that can be known in advance; this and Eqs. (20) and (21) are particularly useful in the derivations presented in Appendices A and B. For the same reason, we define the quantity $\sigma_{f}$ as $\sigma_{f} \equiv \eta k / \omega$ and its 0 subscripted counterpart $\sigma_{\mathrm{f}, 0}$ when referring to pure solvent. 


\section{CALCULATIONS}

As mentioned in the Introduction, the quantity $\Delta D / \Delta f$, referred to as the acoustic ratio, is intimately related with the analyte's intrinsic viscosity, thus providing a crucial link between experimental acoustic measurements and molecular properties. In this Section, we present the way the current model can be employed to predict the acoustic ratio for a given analyte, as well as the input and assumptions necessary for this kind of calculation.

\section{A. Required input}

To profit from the above-presented model, we need to express the quantities involved in Eqs. (16) and (19), in terms of parameters that describe the analyte molecules. The angular frequency is given as a function of the analyte molecular weight by Eq. (A6) in Appendix A. The crystal's own dissipation factor, $D_{s}$, and natural frequency, $\omega_{s}$, as well as the corresponding values referring to operations in liquid, are routinely measured during QCM-D (ring-down) experiments. Alternatively, $\omega_{s}$ can be computed from $\omega_{0}$ (or vice versa) using Eq. (A4). For instance, from actual readings off the machine, we have $D_{0}=1.21 \times 10^{-4}$ and $D_{s}=1.9 \times 10^{-5}$; by inserting these into Eq. (A4), we get $\omega_{s} / \omega_{0}-1=5.1 \times 10^{-5}$; this $\omega_{s} / \omega_{0}$ value is in very good agreement with $(6.0 \pm 1.0) \times 10^{-5}$, an average of directly measured frequency shifts. Using Eqs. (19) and (A6) together with the above values and Eqs. (4), (21), and (A4), the acoustic ratio can be computed, provided that surface coverage (or surface area per analyte molecule, $A / N$ ), viscosity $\eta$ of the solution adjacent to the crystal surface, and the molecule's equivalent hydrodynamic radius $r_{h}^{\text {eq }}$ are known. Apart from parameter optimization in the context of fitting to existing measurements, these quantities can be estimated as described below.

Given the dimensions of anchor points, a minimum surface area per molecule can be hypothesized. For instance, in the case of dsDNA bound on neutravidin protein molecules, the size of neutravidin implies a minimum area per DNA between 40 and $50 \mathrm{~nm}^{2}[22,63]$. We consider a boundary layer of fluid adjacent to the oscillating surface where practically all dissipation takes place. The viscosity, $\eta$ of this layer can be estimated by the commonly employed power-series expression [32,33]

$$
\eta=\eta_{0}\left(1+[\eta] c+K_{H}[\eta]^{2} c^{2}+\cdots\right),
$$

where $[\eta]$ is the analyte's intrinsic viscosity, $K_{H}$ is the Huggins constant, a dimensionless coefficient depending on the nature of both the analyte and the solvent as well as on temperature, and $c$ is the analyte concentration in a control volume that is to be defined. We are going to measure concentration in units of mass per volume so that intrinsic viscosity units will be those of specific volume $\left(\mathrm{cm}^{3} / \mathrm{g}\right)$. To determine the concentration, we note that the acoustic wave travelling through the fluid will almost vanish after a certain distance, so the control volume of interest should be defined as that of a boundary layer containing all strata of fluid parallel to the surface that move at a non-negligible velocity. If we measure the thickness (height) $w \delta$ of this overlayer in units of $\delta$ [ $\equiv 1 / k$, Eq. (4a)], where $w$ is an as yet unknown dimensionless parameter, the concentration in this volume is

$$
c=\frac{M}{N_{\mathrm{A}}(A / N)(w \delta)}=\frac{M k}{N_{\mathrm{A}} w(A / N)},
$$

where $M$ is the analyte's molecular weight and $N_{A}$ is the Avogadro number. The liquid density entering Eq. (4a) can be given by Eq. (A7), although using water density values in subsequent calculations has no discernible effect on the results. Then, if $w$ is known, Eqs. (4a), (22), and (23) can be solved for $c, \eta$, and $k$ as a system of three equations with three unknowns. As shown in Appendix B [Eqs. (B7) and (B10)], the mathematical relation between the acoustic ratio and intrinsic viscosity at the limit of low analyte mass per unit area is given as

$$
\begin{aligned}
-\frac{\Delta D}{\Delta f} \approx \underbrace{\frac{\rho_{0}}{2 w f_{0}}}_{\text {slope }}[\eta] \\
+\underbrace{6 N_{A} \frac{\eta_{0}}{f_{0}^{2}}\left(\lim _{M \rightarrow 0} \frac{r_{h}^{\mathrm{eq}}}{M}\right)+\frac{\frac{1}{w}\left(1-\frac{\rho_{0}}{\rho_{p}}\right)-D_{0}}{f_{0}}}_{\text {intercept }},
\end{aligned}
$$

where $f_{o}$ is the oscillator's frequency when in contact with pure solvent (unloaded) and $\rho_{p}$ is the solute's density. Equation (24) appears to qualitatively describe the experimental data for dsDNA in Refs [22-24]; there is a linear dependence of the acoustic ratio on $[\eta]$ up until a certain molecular weight value and a nonzero intercept. The slope depends on experimental conditions (solvent density and operating frequency) and the unknown parameter $w$. Using the experimentally [24] measured slope $\left(0.34 \times 10^{-9} / \mathrm{Hz}\right)$ at $35 \mathrm{MHz}$, from Eq. (24), we get $w \approx 42.0$; this value is rather unexpected and its meaning will be discussed later.

Finally, before we estimate the equivalent hydrodynamic radius, we note that for the limit in the intercept term of Eq. (24) to be finite and comparable to the experimental value, $r_{h}^{\text {eq }}$ should vary linearly with $M$, at least for low molecular weights - otherwise the intercept would be either zero or infinite (see end of Appendix B for details). By assuming such a linear dependence, we get

$$
r_{h}^{\mathrm{eq}}=K_{r} M \Rightarrow \text { intercept } \approx 6 N_{A} \frac{\eta_{0}}{f_{0}^{2}} K_{r},
$$

where the last term of Eq. (24) is neglected as per the discussion in Appendix B. Using the experimental 
value of $11.2 \times 10^{-9} / \mathrm{Hz}$ for the intercept [24], Eq. (25) gives $K_{r}^{\exp } \approx 0.38 \times 10^{-7} \AA \mathrm{Da}^{-1}$. Such a result implies remarkably small values of equivalent radii (e.g., less than $0.01 \AA$ for $M \approx 100 \mathrm{kDa}$ ). This is not unreasonable because the very definition of the equivalent radius reflects the magnitude of the actual drag forces on the analyte molecules. These forces depend on a particle's velocity relative to the surrounding fluid. As both fluid and particle are entrained by the oscillating surface, they are expected to move almost in phase with each other, resulting in minimal drag (see relevant discussion in Sec. II B). Nevertheless, predicted acoustic ratios are not insensitive to changes in $r_{h}^{\text {eq }}$ because the changes in dissipation and frequency with analyte addition are also very small, especially for low-molecular-weight species, so that the equivalent-radius-dependent term in Eq. (19) presents a non-negligible contribution.

\section{B. Predictions}

The calculated values of $w$ and $r_{h}^{\mathrm{eq}}$ correspond to the low-molecular-weight limit. We are going to test whether acoustic ratio predictions for dsDNA can be extrapolated to high molecular weights by comparing with actual measurements reported in Ref. [24]. In actual QCM-D experiments, a so-called "ring" frequency, $\omega_{r}$, is measured (instead of the natural frequency, $\omega$ ); this is the frequency of the freely decaying oscillations after the external force has been switched off; it depends on the natural frequency and the dissipation factor:

$$
\omega_{r}=\omega \sqrt{1-D^{2} / 4} \text { and } \omega_{r, 0}=\omega_{0} \sqrt{1-D_{0}^{2} / 4}
$$

Then, the acoustic ratio is given by

$$
-\frac{\Delta D}{\Delta f}=-\frac{D-D_{0}}{f_{r}-f_{r, 0}}
$$

where $\omega_{r}=2 \pi f_{r}$ and $\omega_{r, o}=2 \pi f_{r, o}$ for the loaded and solvent cases, respectively. Although Eq. (27) is indeed used in our calculations, the difference between natural and ring frequency is so small that inserting $\omega$ instead of $\omega_{\mathrm{r}}$ has almost no effect on the results.

Apart from the experimental conditions and the crystal's intrinsic properties, the most important factors affecting the acoustic ratio are analyte molecular weight, surface coverage (or surface area per molecule), and analyteequivalent-hydrodynamic radius. We give predictions of the acoustic ratio as a function of molecular weight (base pairs) and intrinsic viscosity, based on our model and estimated input data, as well as the way predictions depend on the equivalent radius or surface coverage. It is known experimentally [28] that for dsDNA,

$$
[\eta]=3.5 \times 10^{-4} \times M^{1.05},
$$

where $[\eta]$ is measured in $\mathrm{ml} / \mathrm{g}$ and $M$ in Da. Using this expression, either the intrinsic viscosity or the molecular weight can serve as input; the same expression can be used to calculate the viscosity from Eq. (22). It will be furthermore assumed that the Huggins constant will be negligible in the range of examined molecular weights (up to approximately 1500 base pairs); this assumption will be revisited later.

Using the iterative scheme of Appendix A and setting $K_{H}=0$ [22,23], we calculate acoustic-ratio predictions for a range of surface-coverage fractions; these are shown in Fig. 2. In particular, we define $100 \%$ coverage as the state of $50-\mathrm{nm}^{2}$ surface area per molecule; thus, $100 \mathrm{~nm}^{2}$ corresponds to $50 \%$ coverage and so on. As previously noted, this limit is a reasonable estimate for dsDNA; neutravidin molecules that are used to bind biotinylated DNA occupy an area of approximately $40 \mathrm{~nm}^{2}$, but they are almost never closely packed on the surface. Furthermore, each neutravidin molecule can accommodate only one dsDNA chain on one of its solvent-facing binding sites due to steric hindrance, hence the above estimated minimum area. In Fig. 3, predicted acoustic ratios for dsDNA are compared to corresponding experimental data as a function of

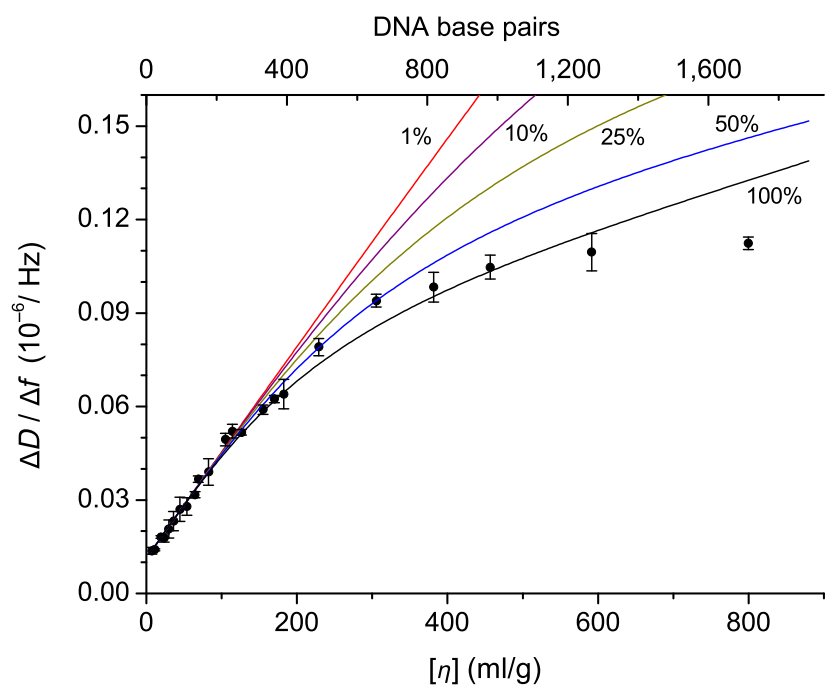

FIG. 2. Acoustic ratio of dsDNA (QCM-D, $35 \mathrm{MHz}$ ) as a function of $[\eta]$ and molecular weight (i.e., number of bp) for different coverage fractions (shown next to each curve), assuming linear dependence of equivalent hydrodynamic radius on molecular weight, $r_{h}^{\text {eq }} \sim M$. An area of $50 \mathrm{~nm}^{2}$ per molecule is taken as the limit of the densest possible coverage, that is, $100 \%$ coverage fraction; its multiples define correspondingly smaller fractions. The remainder of the parameters used in these calculations correspond to the "initial" set in Table I. Experimental points are shown with error bars [24]. 


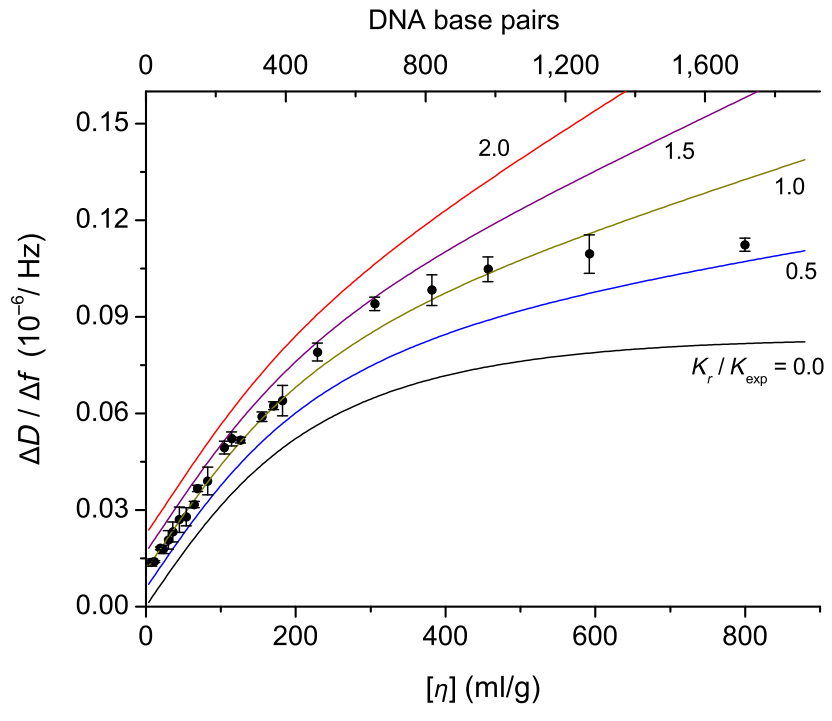

FIG. 3. Acoustic ratio of dsDNA as a function of $[\eta]$ and molecular weight (bp) for different proportionality coefficients $K_{r}$, expressed as multiples of $K_{r}^{\exp }$ (shown next to each curve). The remainder of the parameters used in these calculations correspond to the "initial" set in Table I. Experimental points are shown with error bars [24].

intrinsic viscosity and molecular weight (bp), for a number of values of $K_{r}$, the proportionality constant introduced in Eq. (25). To investigate the effect of deviations from linear dependence of $r_{h}^{\mathrm{eq}}$ on molecular weight, we take as an example a Mark-Houwink-type expression, $r_{h}^{\text {eq }}=$ $K_{r} M^{v}$; in Fig. 4, acoustic-ratio predictions are compared

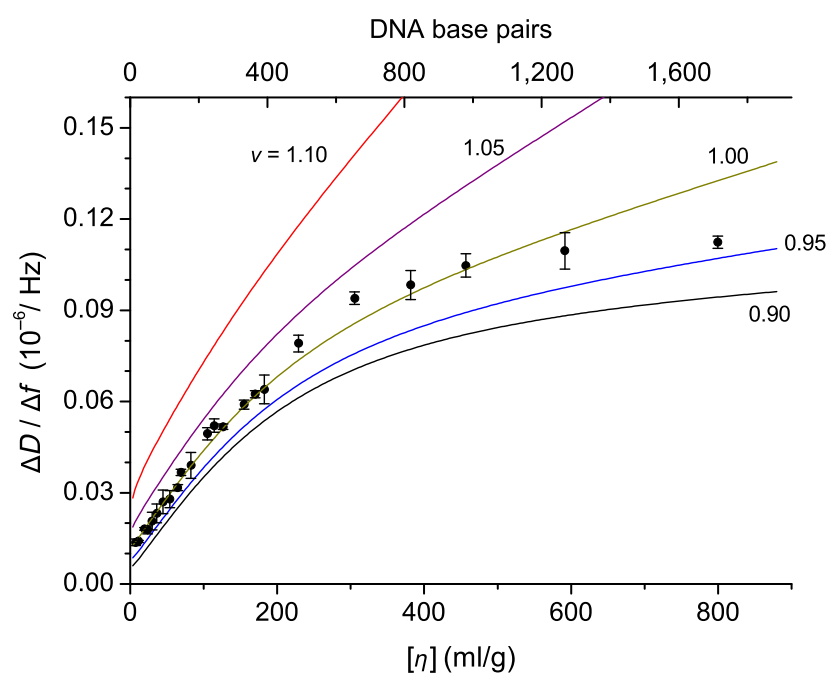

FIG. 4. Acoustic ratio of dsDNA as a function of $[\eta]$ and molecular weight (bp) for different exponents $v$ (shown next to each curve) in a Mark-Houwink type expression, $r_{h}^{\text {eq }}=K_{r} M^{v}$, demonstrating the effect of nonlinear dependence of equivalent radius on molecular weight. The remainder of the parameters used in these calculations correspond to the "initial" set in Table I. Experimental points are shown with error bars [24]. to experiments for a range of exponents $v$ between 0.9 and 1.1 .

\section{DISCUSSION}

The model as presented so far relies on a number of conditions and assumptions that we summarize now for convenience. Apart from the simplifications introduced at the beginning of Sec. II in order to set up a tractable scheme (plane surface, no end-effects, length-preserving linker, and so on), additional approximations are introduced in the course of the ensuing mathematical derivations. In particular, we assume: (a) that the attached particles are free-dangling/floating over the surface, (b) the no-slip condition holds at the solid-liquid interface, (c) thermal noise effects are averaged out, (d) "minimal disturbance" of fluid flow due to the attached particles, (e) drag forces on the analyte particles are adequately described by a Stokes-like term and retarded viscous contributions can be neglected, (f) that the particle's oscillation amplitude is bounded by the surface's oscillation amplitude and gets smaller with distance from the surface (or increasing molecular weight), (g) the particle's oscillation lags by an angle $\varphi$ between zero and $-k y$ with respect to the surface, (h) the inertia of the oscillator in contact with fluid, with $N$ analyte molecules attached on its surface, is adequately approximated as $m \approx m_{s}+\eta_{o} A k_{o} / \omega_{o}+N m_{p}$, (i) "added mass" effects can be neglected because of the small particle acceleration relative to the fluid, $(\mathrm{j})$ dissipation takes place in a boundary fluid layer with a thickness $h$ such that $h / \delta=w$, a parameter to determine, $(\mathrm{k})$ the boundary layer viscosity is given by $\eta=\eta_{o}(1+[\eta] c+\ldots)$ where $c$ is the concentration in this layer, and (1) in the case of dsDNA, $[\eta]$ at the surface is adequately described by the relationship for bulk solutions [Eq. (28)]; this is not required to derive the model equations, but it is useful when comparing theoretical and experimental results.

Given reasonable input parameters, the model then predicts trends that resemble experimental measurements. Data shown in Fig. 2 verify that the acoustic ratio of dsDNA is more or less a linear function of the analyte's intrinsic viscosity, at the limit of low analyte mass per unit surface area. This limit can be attained either by low-molecular-weight species (for which linearity holds regardless of surface coverage and the acoustic ratio is independent of the latter) or by low-coverage fractions (where linearity holds regardless of molecular weight, but the acoustic ratio may strongly depend on coverage). Moreover, a finite intercept is predicted at vanishing intrinsic viscosity, in agreement with experiment. Nonlinearity sets in at about the same range of molecular weights as in experiment, but the curvature is different: a monotonic increase in predicted acoustic ratios seems to persist unlike measured values that appear to converge to a finite limit (plateau). It should be noted that in these calculations, 
TABLE I. Three sets of values for selected model parameters: (a) estimated on the basis of physical arguments and experimental data, (b) optimized, and (c) estimated in the context of an amended model allowing $r_{h}^{\text {eq }}$ to converge to a constant value. Values for the maximum likelihood estimator (MLE) and fractional average absolute error (\%AAE) are also given.

\begin{tabular}{|c|c|c|c|c|c|c|c|}
\hline Parameter & $A / N\left(\mathrm{~nm}^{2}\right)$ & $K_{r} \times 10^{7}$ & $v$ & $w$ & $M_{c}(\mathrm{kDa})$ & MLE & $\% \mathrm{AAE}$ \\
\hline "Initial" & 50 & 0.38 & 1.000 & 42.0 & - & 6.880 & 4.744 \\
\hline "Optimal” & 56.9 & 2.90 & 0.779 & 34.1 & - & 1.945 & 3.625 \\
\hline "Amended" & 73.5 & 0.38 & 0.000 & 33.9 & 124.6 & 1.542 & 3.284 \\
\hline
\end{tabular}

intermolecular interactions are ignored because the Huggins constant and higher terms in expansion Eq. (22) have been set to zero; such terms account for two-body, three-body, and higher interactions.

Before attempting to explain the observed nonlinearity and amend the model to reproduce nonlinear measurements, we look at the exact role of equivalent hydrodynamic radius. In Fig. 3, different proportionality coefficients, $K_{r}$, are tested from zero to twice the value of $K_{r}^{\exp }$. For $K_{r}=0$, the predicted curve passes very close to the origin $(0,0)$, whereas a finite intercept shows up and increases linearly with $K_{r}$. The radius coefficient affects the predicted values over the whole range of examined molecular weights. Higher coefficients, that is, stronger drag forces on the analyte particles, imply higher acoustic ratios. Remarkably, zero-equivalent radius $\left(K_{r}=0\right)$ results in a curve almost parallel to the experimental data set, albeit converging at a lower limit. This evidence indicates that the linear dependence of $r_{h}^{\mathrm{eq}}$ on molecular weight assumed for lighter species need not hold in the range of heavier molecules. The same conclusion can be arrived at if we look at Fig. 4, where a smaller exponent in an assumed Mark-Houwink expression leads to considerably slower increase of acoustic ratio at high molecular weights.

In view of the above, two questions arise: what is the origin of nonlinearity of the acoustic ratio and what is the reason predictions for dsDNA diverge, albeit at a slower rate than the linear part, whereas corresponding experimental measurements tend to converge to a plateau? To answer these questions, we break down the expression for the dissipation factor, Eq. (19), into separate contributions, namely,

$$
\begin{aligned}
\frac{D}{D_{0}}= & \frac{D_{s} / D_{0}}{\omega_{s} / \omega_{0}}+\left(1-\frac{D_{s} / D_{0}}{\omega_{s} / \omega_{0}}\right) \frac{\eta}{\eta_{0}} \frac{k}{k_{0}} \frac{\omega}{\omega_{0}} \\
& +\left(1-\frac{D_{s} / D_{0}}{\omega_{s} / \omega_{0}}\right) \frac{\eta}{\eta_{0}} \frac{6 \pi r_{h}^{\mathrm{eq}}}{(A / N) k_{0}} \frac{\omega}{\omega_{0}} .
\end{aligned}
$$

Using Eq. (29), we compute the acoustic ratio

$$
-\frac{\Delta D}{\Delta \omega_{r}} \equiv-\frac{D-D_{0}}{\omega_{r}-\omega_{r, o}}=-\left(\frac{D_{0}}{\omega_{r, o}}\right) \frac{\left(D / D_{0}-1\right)}{\left(\omega_{r} / \omega_{r, o}-1\right)}
$$

as the sum of two terms:

$$
\begin{aligned}
& \text { "term-A" } \quad-\frac{\Delta D}{\Delta \omega_{\mathrm{vol}}} \\
& =-\left(\frac{D}{\omega_{r, o}}\right) \frac{\left(\frac{D_{s} / D_{0}}{\omega_{S} / \omega_{r, o}}\right)+\left(1-\frac{D_{s} / D_{0}}{\omega_{s} / \omega_{r, o}}\right) \frac{\eta}{\eta_{0}} \frac{k}{k_{0}} \frac{\omega_{r}}{\omega_{r, o}}-1}{\left(\omega_{r} / \omega_{r, o}-1\right)}
\end{aligned}
$$

and

$$
\begin{aligned}
& \text { “term-B" } \quad-\frac{\Delta D}{\Delta \omega_{\mathrm{drag}}} \\
& =-\left(\frac{D}{\omega_{r, o}}\right) \frac{\left(1-\frac{D_{s} / D_{0}}{\omega_{s} / \omega_{r, o}}\right) \frac{\eta}{\eta_{0}} \frac{6 \pi r_{h}^{\mathrm{eq}}}{(A / N) k_{0}} \frac{\omega_{r}}{\omega_{r, o}}}{\left(\omega_{r} / \omega_{r, o}-1\right)} .
\end{aligned}
$$

Both terms, considered as functions of analyte molecular weight, depend on the increase in viscosity $\left(\eta / \eta_{0}\right)$ and mass (through the frequency shift, $\omega_{r} / \omega_{r, 0}$ ), but the first one is modulated by the increase in boundary layer volume (decrease in wavenumber, $k=1 / \delta$ ) which, in turn, affects the concentration, while the second one contains the contribution of drag forces on the analyte particle (via the equivalent hydrodynamic radius $r_{h}^{\mathrm{eq}}$ ).

The two terms and their sum, the total acoustic ratio, are shown in Fig. 5(a) for dsDNA using the same parameter values as in previous calculations (100\% coverage). The volume-dependent term begins with a linear trend and then tends to converge to a limiting value or, at least, increase at a very slow rate beyond a certain cutoff. The drag resistance term starts from a finite value that gives rise to a nonzero intercept, and grows faster with increasing molecular weight or intrinsic viscosity, until becoming the dominant contribution to the predicted monotonic increase of total acoustic ratio. The same two terms are also shown in Fig. 5(b), this time for 1\% coverage. As expected, the radius-dependent term is almost flat because of dividing by the area per molecule, while the volume-modulated term exhibits a nearly perfect linearity reflected in the trend of the total acoustic ratio.

Apart from any constant parts, the two terms differ by the equivalent hydrodynamic radius (over the area per molecule) in "term-B" being in place of the wavenumber in "term-A." Since $r_{h}^{\mathrm{eq}}$ is the only parameter for which an 

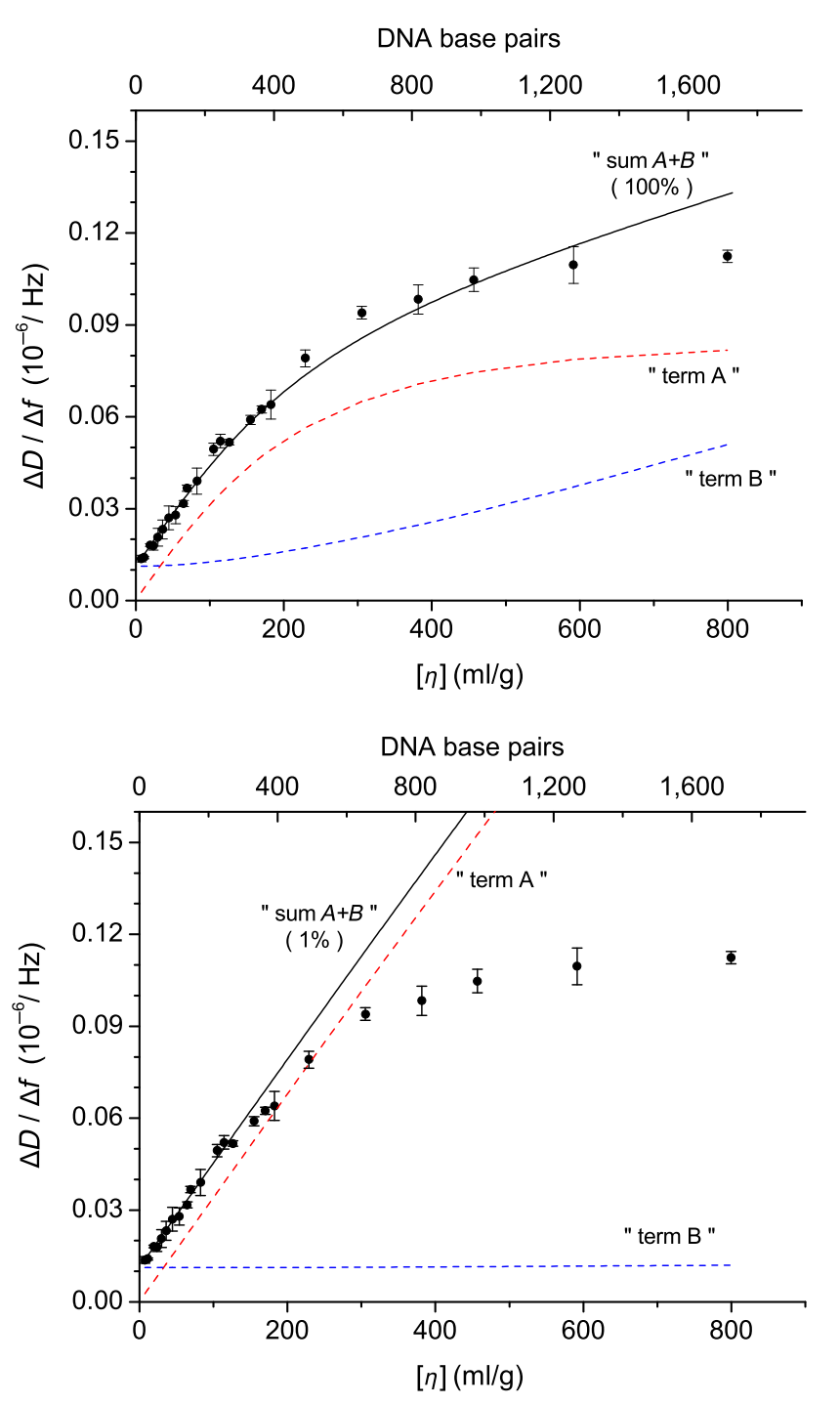

FIG. 5. Predicted acoustic ratio of dsDNA at $100 \%$ and $1 \%$ surface coverage, broken down to a volume-modulated term ("termA") and a contribution from drag forces ("term-B"), Eqs. (31a) and (31b), respectively. The parameters used in these calculations correspond to the "initial" set in Table I. Experimental points are shown with error bars [24].

explicit assumption is made regarding its dependence on molecular weight, it is clear that the particular assumption of linearity is not appropriate except for the lighter species.

We present two alternative routes to improving our model. The first one is merely to relax some of the model parameters relative to their initial values. To this purpose, we optimized the radius coefficient $K_{r}$, exponent $v$, surface area per molecule $A / N$, and boundary layer height $w$, using a maximum likelihood estimator to fit the model to the experimental data, in the context of a global search strategy that combined many local searches starting from different sets of initial values. The Nelder-Mead polytope method [64], as implemented in the Merlin optimization

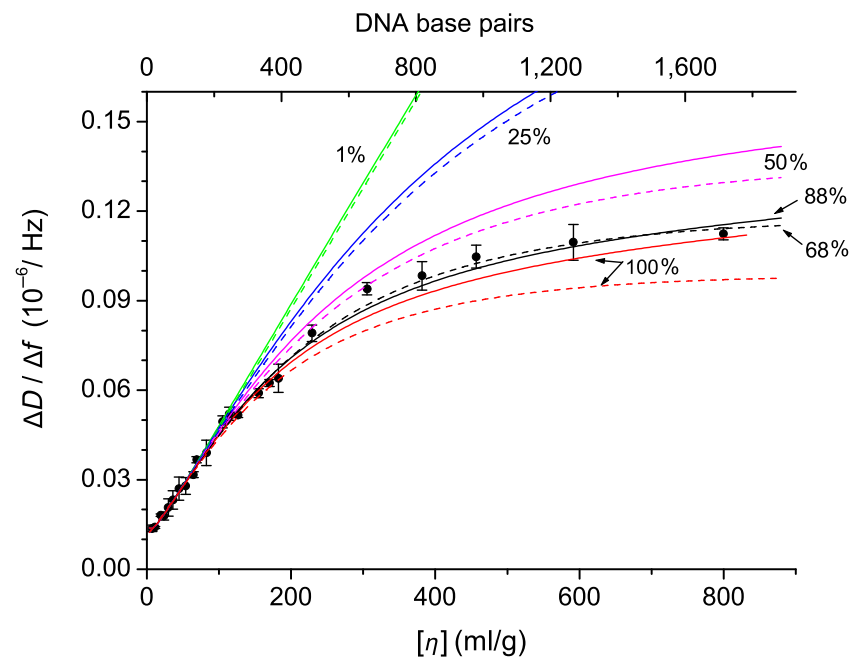

FIG. 6. Acoustic ratio of dsDNA as a function of $[\eta]$ and number of base pairs for different coverage fractions. An area of $50.0 \mathrm{~nm}^{2}$ per molecule is taken as the limit of densest possible coverage, that is, $100 \%$ coverage. The corresponding coverage fractions (\%) are indicated next to each curve. The solid lines are with the "optimal," while the dotted are the "amended" values of Table I. Experimental points are shown with error bars [24].

environment [65], was used in local searches. The initial and optimal values are presented in Table I. As we can see in Fig. 6 (solid lines), considerable improvement has been made, but the predicted acoustic ratio tends to increase rather than converge to a plateau value. Taking an area per molecule of $50 \mathrm{~nm}^{2}$ to indicate full coverage, we can see that $88 \%$ surface coverage is required for an optimal fitting to experimental data.

Notably, the optimized equivalent radius, $r_{h}^{\text {eq }}$, depends sublinearly on molecular weight, which implies a divergent intercept as explained earlier. This observation leads us to the second route, namely, to derive a more general expression for the equivalent radius such that at low molecular weights a linear dependence is restored, whereas larger species exhibit a weaker dependence of $r_{h}^{\text {eq }}$ on molecular weight. We do this by modifying our expression for $r_{h}^{\mathrm{eq}}$ [Eq. (25)] so that it tends to a linear relation for small molecular weights and a sublinear dependence on high molecular weights, namely,

$$
r_{h}^{\mathrm{eq}}=K_{r} \frac{M}{1+\left(\frac{M}{M_{c}}\right)^{1-\nu}} \rightarrow \begin{cases}K_{r} M & \text { when } M \ll M_{c}, \\ K_{r} M_{c}^{1-v} M^{v} & \text { when } M \gg M_{c},\end{cases}
$$

where $M_{c}$ is a characteristic value that governs the transition from linear to sublinear dependence and $v$ can vary between 0 and 1 . Although the above expression is not derived by a rigorous theoretical argument, we expect it to be capable of capturing the actual trends, especially with regards to the limiting cases. The additional parameter $M_{c}$ 
offers a wider margin of flexibility in terms of describing a range of species of varying stiffness.

The coefficient $K_{r}$ is assigned the same value as in our previous calculations. We try $v=0$, which implies convergence of equivalent radius to a finite limit with increasing molecular weight, and optimize the characteristic value $M_{c}$, surface area per molecule $A / N$, and boundary layer height $w$ using the same protocol as previously used. The new parameter values are reported in the last row (amended) of Table I. Results are shown in Fig. 6 (dotted lines); we can see that the new predictions match the trend of the actual data quite closely, but they also suggest a lower optimal surface coverage, namely, $68 \%$.

The deviations of certain experimental points from the predicted curve can be explained in terms of differences in coverage: we do not expect the exact same coverage to be invariably attained in all experiments. The value tried here should be understood as a kind of average. This is, in fact, one more reason why our predictions fail to fully follow the data: using the same surface area per molecule $A / N$ and dimensionless boundary layer height $w$ is a simplification. Calculations (not shown here) based on measurements for 1294-base-pair DNA imply that the surface area per molecule for the particular analyte should be at least $150 \mathrm{~nm}^{2}$, that is, at least twice as large as the abovedetermined optimal value. Here, a much higher acoustic ratio is predicted by our model for such a low coverage. It appears then that combinations of parameters based on individual experiments rather than a priori assumptions could lead to better fitting. Such calculations, to be presented in forthcoming publications, will be required to determine the capabilities and limitations of our model.

In Fig. 7, the above-determined optimal area of $73.5 \mathrm{~nm}^{2}$ per molecule is used as a basis corresponding to $100 \%$ coverage and the acoustic ratio of dsDNA is shown for coverage fractions down to $1 \%$ for an assortment of base pair numbers. This is another way of presenting results such as those shown in Figs. 2 and 6. The calculated acoustic ratio appears almost flat for up to approximately $300 \mathrm{bp}$, in apparent agreement with experimental measurements. From that chain length upward, an ever steeper increase of the ratio can be observed with decreasing coverage. Although a monotonic dependence has been observed for particular cases of proteins [27], it has not so far been observed for dsDNA, which shows a remarkable stability $[22,23,25,26]$. We must draw attention here to the fact that the difference in $\Delta \mathrm{D} / \Delta f$ calculated at approximately $0 \%$ and approximately $100 \%$ coverage becomes experimentally discernible (i.e., is $\geq 10 \%$ ) only for DNA with more than approximately $500 \mathrm{bp}$; unfortunately, DNA of such lengths reaches the observed plateau [24] making it difficult to verify this prediction experimentally.

Another open question concerns the role of the parameter $w$. As it turns out, defining a pertinent volume is not a trivial task. The $w$ values obtained/required (approximately

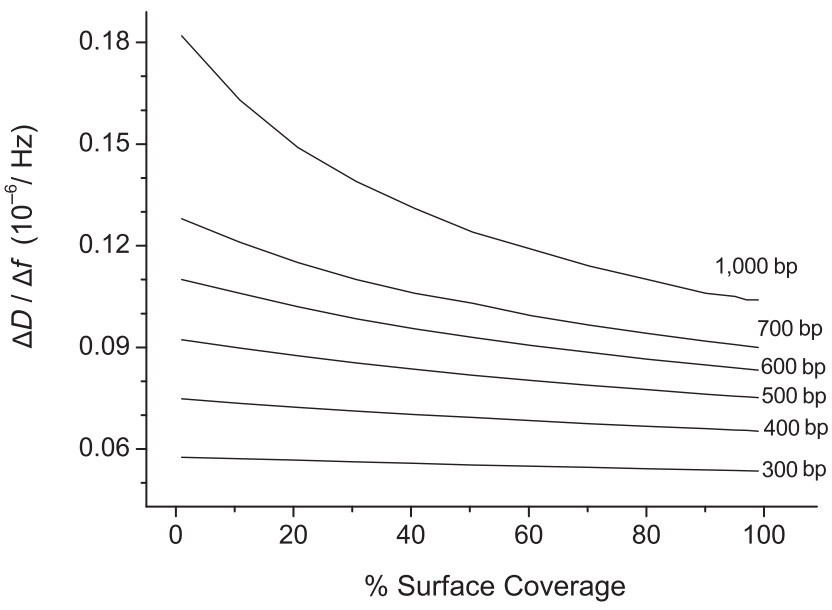

FIG. 7. Predicted acoustic ratio of dsDNA for indicative numbers of bp within the experimentally studied range as a function of surface coverage using the modified model discussed in Sec. IV. Here, the optimal area of $73.5 \mathrm{~nm}^{2}$ per molecule is taken as a measure of $100 \%$ coverage. The remainder of the parameters used in these calculations are from the "amended" set in Table I.

40) to fit the experimental data are clearly beyond the highest plausible value of $w \sim 3$, which would correspond to a complete-decay depth for a liquid-penetrating acoustic wave (where approximately $99 \%$ of its energy is lost). Currently, we lack an explanation for this result, but we call attention to the finding that $w$ only controls the slope of the curve and not its linearity. The existence of another component/parameter that affects this slope but is unaccounted for by the current form of our model cannot be excluded.

We close this section with a brief discussion on the usability of our model with respect to raw experimental data for a given analyte that is adsorbed in the form of free-dangling molecules attached to discrete binding points. It has already been verified experimentally [24] that the linear part of the acoustic ratio dependence on intrinsic viscosity has a rather universal validity, provided the same substrate (e.g., neutravidin) is used. Then, one can readily determine the intrinsic viscosity of the analyte from its acoustic ratio and, given its molecular weight, draw important conclusions about the molecule's shape under the experimental conditions. For example, having a polymer in loop/train or mushroom or brush configurations would result in entirely different $\Delta D / \Delta f$ ratios because of different $[\eta]$ values; something similar has been recently verified in the case of a "globe + coil" protein molecule [27]. Fitting our model to the sensor readings will add more information of theoretical and practical interest. Such information includes surface coverage with time and the extent to which drag forces participate in the overall dissipation mechanism. The latter is quantified in the form of equivalent radius values that we expect to be related with the linker's and analyte molecule's stiffnesses (see also discussion at the end of Appendix B). Application of 
TABLE II. List of model parameters; given a set of $D, f$ (or acoustic ratio) measurements, these parameters are used to calculate all other quantities discussed in the text. When studying a homologous series of analytes, the number of unknown parameters can be reduced by replacing some of them by Mark-Houwink or other convenient expressions as in Eqs. (25) and (32); the parameters of these expressions are then to be determined.

\begin{tabular}{|c|c|c|}
\hline Symbol & Meaning & Determination \\
\hline$D_{s}$ & $\begin{array}{l}\text { Crystal's own dissipation coefficient at the } \\
\text { overtone of interest }\end{array}$ & Measured by the device operating in the air. \\
\hline$D_{0}$ & $\begin{array}{l}\text { Dissipation coefficient in the presence of pure } \\
\text { solvent at the overtone of interest }\end{array}$ & Measured at the beginning of an experiment. \\
\hline$\omega_{s}=2 f_{s}$ & $\begin{array}{l}\text { Crystal's own natural angular frequency at the } \\
\text { overtone of interest }\end{array}$ & $\begin{array}{l}\text { Measured by the device operating in the air; } \\
\text { also related with } D_{s}, D_{0} \text {, and } \omega_{0} \text { via } \\
\text { Eq. (A4) of Appendix A. }\end{array}$ \\
\hline$\omega_{0}=2 \pi f_{0}$ & $\begin{array}{l}\text { Natural angular frequency in the presence of } \\
\text { pure solvent at the overtone of interest }\end{array}$ & $\begin{array}{l}\text { Measured at the beginning of experiments; } \\
\text { also related with } D_{s}, D_{0} \text {, and } \omega_{s} \text { via Eq. (A4) } \\
\text { of Appendix A. }\end{array}$ \\
\hline$A / N$ & $\begin{array}{l}\text { Crystal surface area per attached analyte } \\
\text { molecule }\end{array}$ & $\begin{array}{l}\text { At the limit of full coverage, it can be assumed } \\
\text { (e.g.. approximately } 50 \mathrm{~nm}^{2} \text { for a } \\
\text { neutravidin substrate); otherwise it will be } \\
\text { fitted to measurements. }\end{array}$ \\
\hline$\eta_{0}$ & Pure solvent viscosity & $\begin{array}{l}\text { Water's viscosity suffices for typical buffer } \\
\text { solutions. }\end{array}$ \\
\hline$M$ & Analyte's molecular weight & If unknown, it can be fitted to measurements. \\
\hline$[\eta]$ & Analyte's intrinsic viscosity & If unknown, it can be fitted to measurements. \\
\hline$K_{H}$ & $\begin{array}{l}\text { Analyte's Huggins coefficient at given } \\
\text { conditions (solvent, temperature) }\end{array}$ & If unknown, it can be fitted to measurements. \\
\hline$r_{h}^{\mathrm{eq}}$ & Analyte's equivalent hydrodynamic radius & It must be fitted to measurements. \\
\hline$\rho_{p}$ & Analyte particle's density & $\begin{array}{l}\text { It has little effect on overall results; if it is not } \\
\text { given, an approximate estimate can be used } \\
\text { to reduce the number of unknown } \\
\text { parameters. }\end{array}$ \\
\hline$w$ & $\begin{array}{l}\text { Boundary layer height expressed as multiple of } \\
\text { penetration length }\end{array}$ & It must be fitted to measurements. \\
\hline
\end{tabular}

our model to individual experiments as well as elucidation of the role of linker and analyte flexibility on acoustic measurements and model predictions are the subject of ongoing work in our laboratory and will be discussed in detail in forthcoming publications. For the reader's convenience, all parameters pertinent to application of our model are presented in Table II.

\section{CONCLUSIONS}

We present a theoretical model of the QCM-D acoustic device operating in liquid with discretely tethered analyte molecules dangling away from its surface. The model combines the mechanisms of surface-fluid viscous coupling (continuous) and hydrodynamic drag forces on individually attached particles (discrete). Its derivation relies on certain simplifying assumptions to transform the equations of motion of the oscillating surface and tethered molecules to a single equation akin to a damped harmonic oscillator. This equation can be mapped to the standard form with coefficients given in terms of the angular frequency, $\omega$, and dissipation factor, $D$. By such a mapping, we can express these two quantities as functions of analyte and other system properties that enter the initial equations. Then, it is straightforward to calculate the change in dissipation $\Delta D$, frequency shift $\Delta f$, and their ratio (acoustic ratio) for a variety of input conditions including operating frequency and surface coverage, but also analyte properties such as intrinsic viscosity and an introduced parameter, herein termed equivalent hydrodynamic radius. The latter reflects the drag forces due to the particles moving slightly out of phase with respect to the surrounding fluid.

It is verified by theoretical analysis (Appendix B) and numerical calculations that the model is consistent with the discrete binding theory proposed by Tsortos [22-24] about the linear dependence of the acoustic ratio on the analyte intrinsic viscosity. The latter is recovered as a special case at the limit of low analyte mass concentration either because of low analyte molecular weight (regardless of surface coverage) or because of low surface coverage (regardless of analyte molecular weight). Using estimates for the model parameters based on physical reasoning, it is verified that the model also reproduces other features of experimental measurements not predicted by the original theory, namely, a nonzero intercept of the acoustic ratio for dsDNA with zero intrinsic viscosity and 
nonlinear dependence for dsDNA beyond a certain molecular weight. By splitting the acoustic ratio into two contributions, it is found that its exact behavior depends largely on the part containing the drag resistance term as quantified by the equivalent hydrodynamic radius. Its existence is manifested in the nonzero intercept and its dependence on molecular weight (or intrinsic viscosity) has a large impact on the predicted behavior at high molecular weights. By appropriately tuning this contribution, it is possible to not only match the experimental results, but also to estimate the average surface coverage at about $75 \mathrm{~nm}^{2}$ per dsDNA molecule.

Apart from assigning the parameters plausible guess values as in Sec. III, it is also possible to optimize the model by means of numerical techniques. An example of such calculations is briefly presented in the discussion about the dependence of $r_{h}^{\text {eq }}$ on molecular weight in the previous Section. We have carried out more calculations of this kind (not shown) by fitting a number of other parameters as well. It is found that their optimal values are close to the ones employed in Sec. III; among others, the Huggins constant is negligible, indicating very weak particle-particle interactions.

One limitation should be noted here regarding the assumption of common average values of certain parameters for all the species studied (surface area per molecule, height parameter $w$ ); this is not an insurmountable obstacle though. The equations for the dissipation factor and frequency shift can be adapted to work for sets of raw data obtained directly from acoustic experiments for a single species. Typically, a large number of data points is obtained from such experiments; this is an ideal setting for model fitting involving a relatively small set of parameters, unlike the case of acoustic ratios that requires many experiments over a range of molecular weights. In fact, the main reason why the latter route is chosen in this work is to verify and extend the previous theory of discrete binding by demonstrating consistency with available measurements.

Other open questions include the introduction of an explicit term for the particle-surface linker stiffness, the effect of thermal noise, and possibly an extension to models where the analyte molecules are endowed with internal structure, for example, multibead or flexible rodlike particles. As the model complexity grows, though, we are brought closer to numerical methods of particle dynamics simulations. Using our model as a means to tune and initialize the parameters in more sophisticated simulations is certainly an option.

\section{ACKNOWLEDGMENTS}

We acknowledge the European Commission for financially supporting this work through Research Innovation HORIZON2020 - ICT 20-2015 LoveFood2Market Grant
No. 687681 and FET-OPEN-2015 CATCH-U-DNA Grant No. 737212 .

\section{APPENDIX A: EXPRESSIONS FOR THE FREQUENCY SHIFT AND NUMERICAL PROCEDURES TO CALCULATE VISCOSITY, CONCENTRATION, AND DENSITY}

\section{Frequency shifts}

We derive expressions for the frequency shift from air to solvent and solvent to solution. The angular frequency of oscillations in the air is

$$
\omega_{s}=\sqrt{\frac{K}{m_{s}}} .
$$

When we add pure solvent, the additional inertia of fluid entrained by the oscillating surface should be accounted for:

$$
\omega_{0}=\sqrt{\frac{K}{m_{s}+\frac{\eta_{0} k_{0} A}{\omega_{0}}}}=\sqrt{\frac{K / m_{s}}{1+\frac{\eta_{0} k_{0} A}{\omega_{0} m_{s}}}}=\frac{\omega_{s}}{\sqrt{1+\frac{\eta_{0} k_{0}}{\omega_{0}} \frac{1}{\sigma_{s}}}} .
$$

If we combine the above with Eq. (20) or (21) of the main text for the parameter $\sigma_{s}$ we find

$$
\omega_{0}=\frac{\omega_{s}}{\sqrt{1+\frac{D_{0} \omega_{0}-D_{s} \omega_{s}}{\left(1-D_{0}\right) \omega_{0}}}},
$$

which is transformed to the following second-order polynomial equation

$$
\omega_{0}^{2}-D_{s} \omega_{s} \omega_{0}-\left(1-D_{0}\right) \omega_{s}^{2}=0 .
$$

By rearranging Eq. (A3), we obtain the following corollary that can be of use when trying to write certain derivations in a more compact form:

$$
\frac{\omega_{s}^{2}}{\omega_{0}^{2}}=\frac{1-D_{s} \omega_{s} / \omega_{0}}{1-D_{0}}
$$

By solving the second-order equation for $\omega_{s}$, we find

$$
\omega_{s}=\frac{-D_{s}+\sqrt{D_{s}^{2}+4\left(1-D_{0}\right)}}{2\left(1-D_{0}\right)} \omega_{o} .
$$

Inserting values of $D_{0}=1.21 \times 10^{-4}$ and $D_{s}=1.9 \times 10^{-5}$, which are averages of routine measurements taken during our experiments (QCM-D, $35 \mathrm{MHz}$ ), we compute $\omega_{s} / \omega_{0}-1=5.1 \times 10^{-5}$, in good agreement with $(6.0 \pm 1.0) \times 10^{-5}$, an average based on corresponding frequency readings. 
When analyte is introduced, its mass minus the mass of displaced solvent is added to the crystal plus entrained fluid's inertia according to the approximate expression used in conjunction with Eq. (14a). Then

$$
\begin{aligned}
\omega & =\sqrt{\frac{K}{m_{s}+\frac{\eta_{0} k_{0} A}{\omega_{0}}+\frac{N M}{N_{A}}}}=\sqrt{\frac{K / m_{s}}{1+\frac{\eta_{0} k_{0} A}{\omega_{0} m_{S}}+\frac{N M}{N_{A} m_{S}}}} \\
& =\frac{\omega_{S}}{\sqrt{1+\frac{\eta_{0} k_{0}}{\omega_{0}} \frac{1}{\sigma_{S}}+\frac{N M}{N_{A} m_{s}}}}
\end{aligned}
$$

where $M$ is the analyte molecular weight and $N_{A}$ is the Avogadro number. If we combine the above with Eq. (20) or (21) of the main text for the parameter $\sigma_{s}$, we find

$$
\omega=\frac{\omega_{s}}{\sqrt{1+\frac{D_{0}-D_{s} \omega_{s} / \omega_{0}}{1-D_{0}}+\frac{N M}{N_{A} m_{s}}}} .
$$

We can write this in a more compact manner if we take advantage of Eq. (A3a):

$$
\omega=\frac{\omega_{0}}{\sqrt{1+\frac{M}{N_{A}\left(\frac{\omega_{s}}{\omega_{0}}\right)^{2} \frac{m_{s}}{N}}}},
$$

where we express the result as a function of $m_{s} / N$ to be reminded of its connection with surface area per molecule $A / N$, via Eq. (20).

\section{Calculation details}

The equations derived so far can serve to calculate the angular frequency given the other quantities they depend on, including the fluid density entering Eq. (4a). Although it is shown in practice that use of the solvent density will not substantially alter the results, a simple expression for the solution density is derived for the sake of completeness. Let us consider our reference volume $V=w \delta A$ that we use to define concentration. Then, we can write

$$
c=\frac{N m_{p}}{V}=\frac{m_{w}+N m_{p}}{V}-\frac{m_{w}}{V},
$$

where $m_{w}$ is the mass of the solvent occupying the reference volume apart from its part occupied by the analyte (i.e., $\rho_{0} V-N \rho_{0} m_{p} / \rho_{p}$ ), therefore,

$$
\begin{aligned}
c & =\frac{m_{w}+N m_{p}}{V}-\frac{m_{w}}{V}=\rho-\frac{\rho_{0} V-N \frac{\rho_{0}}{\rho_{p}} m_{p}}{V} \\
& =\rho-\rho_{0}+N \frac{\rho_{0}}{\rho_{p}} \frac{m_{p}}{V}=\rho-\rho_{0}+\frac{\rho_{0}}{\rho_{p}} c,
\end{aligned}
$$

or

$$
\rho=\rho_{0}+\left(1-\frac{\rho_{0}}{\rho_{p}}\right) c .
$$

This analysis considers only solvent displacement, but it is possible to extend it to account for solvation effects as well. Using similar mass balance arguments, we derive

$$
\rho=\rho_{0}+\left(1-\frac{v}{v_{w}}+\frac{v_{h}}{v_{w}}\right) c .
$$

where $v$ and $v_{w}$ are the specific molecular volumes of analyte and solvent, respectively, and $v_{h}$ serves to quantify the corresponding contribution from analyte solvation.

A simple iterative scheme suffices to solve the required set of equations very quickly (usually less than ten iterations) and at any desired accuracy (a fractional error tolerance of $10^{-15}$ is used in our calculations); this is summarized in the following steps:

Step 0. (initializations). Input consists of $A / N, \eta_{0}, K_{H}, M$, $m_{s} / N, \rho_{0}, \rho_{p}, w, \omega_{0}, \omega_{s}$ and the desired convergence tolerance. Of these quantities, $A / N$ and $K_{H}$ can be either user-defined estimates or trial values occurring during a fitting numerical calculation; $m_{\mathrm{s}}$ is calculated from Eq. (21). Use Eq. (A6) to calculate $\omega$, set $\rho=\rho_{0}$ and $\eta=\eta_{0}$, and initialize $k=k_{0}=\left(0.5 \omega_{0} \rho_{0} / \eta_{0}\right)^{0.5}$. Save initial values of $c, \eta, k, \rho$ as "old values."

Step 1. Use Eq. (23) to calculate concentration.

Step 2. Use Eq. (A7) to calculate density.

Step 3. Use Eq. (22) to calculate viscosity.

Step 4. Calculate new wavenumber $k=(0.5 \omega \rho / \eta)^{0.5}$, [Eq. (4a)].

Step 5. Check convergence by comparing current viscosity, density, concentration, and wavenumber with "old" values; on convergence or maximum allowed number of iterations, Exit.

Step 6. Update old values of $c, \eta, \rho$, and $k$ and return to Step 1.

\section{APPENDIX B: APPROXIMATE EXPRESSIONS}

We show that at the limit of low analyte mass (either due to sparse coverage or due to low analyte molecular weight), the acoustic ratio exhibits linear dependence on intrinsic viscosity; also, a nonzero intercept is obtained, which is intimately related with the equivalent hydrodynamic radius. To facilitate the derivations, we ignore the difference between ring-down and natural frequency and approximate the acoustic ratio as follows:

$$
-\frac{\Delta D}{\Delta f} \equiv-\frac{\left(D-D_{0}\right)}{\left(f-f_{0}\right)}=-\frac{D_{0}}{f_{0}} \frac{\left(D / D_{0}-1\right)}{\left(\omega / \omega_{0}-1\right)} .
$$

Also, in order to avoid complicated expressions, we neglect the crystal's internal friction, that is, we set $D_{s}=0$; this is legitimate because its contributions do not play a substantial role in the derivations. We note, though, 
that the same results can be arrived at without such a simplification (calculations not shown). Now, frequencies will be replaced by their angular counterparts until the final results, where multiplication by $2 \pi$ will take place. By inserting Eq. (19) and applying our approximations, we write

$$
-\frac{\Delta D}{\Delta \omega}=-\frac{D_{0}}{\omega_{0}} \frac{\frac{\eta}{\eta_{0}}\left(\frac{k}{k_{0}}+\frac{6 \pi r_{h}^{\mathrm{eq}}}{(A / N) k_{0}}\right) \frac{\omega}{\omega_{0}}-1}{\omega / \omega_{0}-1} .
$$

If we write $\omega / \omega_{o}=\left(1+\omega / \omega_{o}-1\right)$ and rearrange, the above can be broken down to

$$
\begin{aligned}
-\frac{\Delta D}{\Delta \omega}= & \underbrace{-\frac{D_{0}}{\omega_{0}} \frac{\frac{\eta}{\eta_{0}}\left(\frac{k}{k_{0}}+\frac{6 \pi r_{h}^{\mathrm{eq}}}{(A / N) k_{0}}\right)-1}{\omega / \omega_{0}-1}}_{\text {term } 1} \\
& -\underbrace{\frac{D_{0}}{\omega_{0}} \frac{\eta}{\eta_{0}}\left(\frac{k}{k_{0}}+\frac{6 \pi r_{h}^{\mathrm{eq}}}{(A / N) k_{0}}\right)}_{\text {term } 2} .
\end{aligned}
$$

In the following, we consider the limit of very low concentrations that can be attained by sparse surface coverage, low molecular weights, or both. First, we deal with "term2 " in Eq. (B2) by noting that at low concentrations, $\eta$ $\rightarrow \eta_{0}, k \rightarrow k_{0}$, and $r_{h}^{\mathrm{eq}}$ vanishes, so this term tends to $-D_{0} / \omega_{0}$. In the remainder of our analysis, we focus on "term-1"

$$
-\frac{\Delta D}{\Delta \omega}=\underbrace{-\frac{D_{0}}{\omega_{0}} \frac{\frac{\eta}{\eta_{0}}\left(\frac{k}{k_{0}}+\frac{6 \pi r_{h}^{\mathrm{eq}}}{(A / N) k_{0}}\right)-1}{\omega / \omega_{0}-1}}_{\text {term } 1}+\text { “term 2". }
$$

Using $2 k^{2} \equiv \omega \rho / \eta$, we rewrite the numerator of "term- 1 " as

$$
D_{0}\left(\left(\frac{\omega \rho \eta}{\omega_{0} \rho_{0} \eta_{0}}\right)^{1 / 2}+\frac{6 \pi r_{h}^{\mathrm{eq}}}{(A / N) k_{0}} \frac{\eta}{\eta_{0}}-1\right) .
$$

Finally, we write an approximate expression for the fractional change in angular frequency, taking the low concentration assumption into account. We start with Eq. (A6) shown in Appendix A and write

$$
\frac{\omega}{\omega_{0}}-1=\frac{1}{\sqrt{1+\frac{M / N_{A}}{\frac{\omega_{s}^{2}}{\omega_{0}^{2}}}} \frac{m_{s}}{N}}-1
$$

Using the parameter $\sigma_{s}$ defined in Eqs. (20) and (21) and combining with Eq. (23), we can rewrite this in terms of analyte concentration

$$
\frac{\omega}{\omega_{0}}-1=\frac{1}{\sqrt{1+\frac{w}{\frac{\omega_{s}^{2}}{\omega_{0}^{2}} c} c}}-1 .
$$

We take its Taylor expansion: at the limit of very low concentration, frequency shifts are so small that it suffices to truncate the inverse square to first order, $(1+x)^{-1 / 2} \approx$ $1-x / 2$,

$$
\frac{\omega}{\omega_{0}}-1 \approx-\frac{w}{2 \frac{\omega_{s}^{2}}{\omega_{0}^{2}} \sigma_{s} k} c .
$$

If we introduce the definition of $\sigma_{\mathrm{s}}$ [Eqs. (20) and (21)] and the expression for the wavenumber $2 k_{0}^{2}=\omega_{0} \rho_{0} / \eta_{0}$ and rearrange,

$$
\frac{\omega}{\omega_{0}}-1 \approx-\frac{D_{0}}{1-D_{0}} \frac{w}{\rho_{0} \frac{\omega_{s}^{2}}{\omega_{0}^{2}} \frac{k}{k_{0}}} c \approx-D_{0} \frac{w}{\rho_{0} \frac{\omega_{s}^{2}}{\omega_{0}^{2}} \frac{k}{k_{0}}} c .
$$

Now, we can use our results so far to estimate the acoustic ratio under the conditions of vanishingly small concentrations; after a few more operations we find

$$
-\frac{\Delta D}{\Delta \omega} \approx-\frac{D_{0}}{\omega_{0}} \frac{\left(\frac{\omega \rho \eta}{\omega_{0} \rho_{0} \eta_{0}}\right)^{1 / 2}+\frac{6 \pi r_{h}^{\mathrm{eq}}}{(A / N) k_{0}} \frac{\eta}{\eta_{0}}-1}{-D_{0} \frac{w}{\rho_{0} \frac{\omega_{s}^{2} k}{\omega_{0}^{2}} c} c}+\text { "term 2", }
$$

or (using $\left.2 k^{2}=\omega \rho / \eta\right)$

$$
-\frac{\Delta D}{\Delta \omega} \approx \frac{\rho_{0}}{w \omega_{0}} \frac{\frac{\omega \rho}{\omega_{0} \rho_{0}}+\left(\frac{\omega \rho}{\omega_{0} \rho_{0}}\right)^{1 / 2} \frac{6 \pi r_{h}^{\mathrm{eq}}}{(A / N) k_{0}}\left(\frac{\eta}{\eta_{0}}\right)^{1 / 2}-\left(\frac{\omega \rho}{\omega_{0} \rho_{0}}\right)^{1 / 2}\left(\frac{\eta}{\eta_{0}}\right)^{-1 / 2}}{c}+\text { "term } 2 " .
$$

Then, we expand the viscosity fractions using Eq. (22), truncating the series where appropriate and rearranging to find 


$$
\begin{aligned}
-\frac{\Delta D}{\Delta \omega} \approx & \frac{\rho_{0}}{2 w \omega_{0}}\left(\frac{\omega \rho}{\omega_{0} \rho_{0}}\right)^{1 / 2} \frac{[\eta] c+K_{H}[\eta]^{2} c^{2}+\cdots}{c} \\
& +\underbrace{\frac{\rho_{0}}{w \omega_{0}}\left(\frac{\omega \rho}{\omega_{0} \rho_{0}}\right)^{1 / 2} \frac{6 \pi r_{h}^{\mathrm{eq}}}{(A / N) k_{0}}\left(1+\frac{1}{2}[\eta] c+\frac{1}{2} K_{H}[\eta]^{2} c^{2}+\cdots\right)+\frac{\frac{\omega \rho}{\omega_{0} \rho_{0}}-\left(\frac{\omega \rho}{\omega_{0} \rho_{0}}\right)^{1 / 2}}{c}+\text { "term 2" }}_{\text {"Intercept" }},
\end{aligned}
$$

or

$$
\begin{aligned}
-\frac{\Delta D}{\Delta \omega} \approx & \frac{\rho_{0}}{2 w \omega_{0}}\left(\frac{\omega \rho}{\omega_{0} \rho_{0}}\right)^{1 / 2}[\eta]\left(1+K_{H}[\eta] c+\cdots\right) \\
& + \text { "intercept". }
\end{aligned}
$$

At the limit of very low concentrations, $\omega / \omega_{0}$ and $\rho / \rho_{0}$ tend to 1 , the concentration dependent terms tend to vanish, and we obtain the result

$$
-\frac{\Delta D}{\Delta \omega} \approx \underbrace{\frac{\rho_{0}}{2 w \omega_{0}}}_{\text {"slope" }}[\eta]+\underbrace{\left.\left(-\frac{\Delta D}{\Delta \omega}\right)\right|_{M=0}}_{\text {"intercept" }} .
$$

This is quite reminiscent not only of our "discrete binding theory" [22-24] predicting linear dependence on intrinsic viscosity, but also of measurements for dsDNA where the acoustic ratio is found to vary linearly with $[\eta]$ for $M$ up to approximately $350 \mathrm{kDa}$ (i.e., $500-600 \mathrm{bp}$ ) and having a nonzero intercept at the limit of vanishing molecular mass. Notably, the slope in Eq. (B7) depends only on the operating conditions (solvent density, oscillation frequency) and the unknown dimensionless height parameter $w$.

Now, we turn our attention to the "intercept" terms that were neglected so far and look at their behavior with vanishing intrinsic viscosity, which also implies very low molecular weight and correspondingly low concentration. We calculate the limit of the "intercept" part of Eq. (B6) under such conditions (frequencies replace angular frequencies after multiplying both sides by $2 \pi$ ),

$$
-\left.\frac{\Delta D}{\Delta \omega}\right|_{\mathrm{M}=0}=\lim _{M \rightarrow 0}\left[\frac{\rho_{0}}{w \omega_{0}}\left(\frac{\omega \rho}{\omega_{0} \rho_{0}}\right)^{1 / 2} \frac{6 \pi r_{h}^{\mathrm{eq}}}{(A / N) k_{0}}\left(1+\frac{1}{2}[\eta] c+\frac{1}{2} K_{H}[\eta]^{2} c^{2}+\cdots\right)+\frac{\frac{\omega \rho}{\omega_{0} \rho_{0}}-\left(\frac{\omega \rho}{\omega_{0} \rho_{0}}\right)^{1 / 2}}{c}+\right.\text { "term 2"] }
$$

or

$$
\begin{aligned}
-\left.\frac{\Delta D}{\Delta f}\right|_{\mathrm{M}=0}= & \frac{\rho_{0}}{w f_{0}}\left[\lim _{M \rightarrow 0} \frac{6 \pi r_{h}^{\mathrm{eq}}}{(A / N) k_{0}}+\lim _{M \rightarrow 0} \frac{\left(\frac{\omega \rho}{\omega_{0} \rho_{0}}\right)^{1 / 2}-1}{c}\right] \\
& -\frac{D_{0}}{f_{0}} .
\end{aligned}
$$

To calculate the first limit in the above result, we use $c=N M k /\left(N_{A} w A\right)$ and $k_{0}^{2}=\pi f_{0} \rho_{0} / \eta_{0}$, and we find

$$
\frac{\rho_{0}}{w f_{0}} \lim _{M \rightarrow 0} \frac{6 \pi r_{h}^{\mathrm{eq}}}{(A / N) k_{0}}=6 N_{A} \frac{\eta_{0}}{f_{0}^{2}}\left(\lim _{M \rightarrow 0} \frac{r_{h}^{\mathrm{eq}}}{M}\right) .
$$

To calculate the second limit, we ignore the fractional change in frequency because it should be very small in comparison with the fractional change in boundary layer density. Indeed, by Eq. (A7), the latter is of the order of $c / \rho_{0}$, whereas the former is of the order of $w c \delta / \sigma_{s}$, as shown by the derivations in previous paragraphs, which is found to be orders of magnitude smaller when using values typically measured at $35 \mathrm{MHz}$. Then, the sought limit is approximated as:

$$
\frac{\rho_{0}}{w f_{0}} \lim _{M \rightarrow 0} \frac{\left(\frac{\omega \rho}{\omega_{0} \rho_{0}}\right)^{1 / 2}-1}{c} \approx \frac{\rho_{0}}{w f_{0}} \lim _{M \rightarrow 0} \frac{\left(\frac{\rho}{\rho_{0}}\right)^{1 / 2}-1}{c},
$$

and combining with Eq. (A7) and taking Taylor expansions justified by the low concentration assumption, we find

$$
\frac{\rho_{0}}{w f_{0}} \lim _{M \rightarrow 0} \frac{\left(\frac{\omega \rho}{\omega_{0} \rho_{0}}\right)^{1 / 2}-1}{c} \approx \frac{1}{w f_{0}}\left(1-\frac{\rho_{0}}{\rho_{p}}\right)
$$


We find the intercept by inserting the results in Eq. (B7) or

$$
-\left.\frac{\Delta D}{\Delta f}\right|_{\mathrm{M}=0}=6 N_{A} \frac{\eta_{0}}{f_{0}^{2}}\left(\lim _{M \rightarrow 0} \frac{r_{h}^{\mathrm{eq}}}{M}\right)+\frac{\frac{1}{w}\left(1-\frac{\rho_{0}}{\rho_{p}}\right)-D_{0}}{f_{0}} .
$$

Generally, if $r_{h}^{\text {eq }}$ increases at a faster than linear rate with molecular weight, $M$, a zero- $M$ limit equal to infinity is implied by Eq. (B10); conversely, if it varies at a slower than linear rate, the limit of $r_{h}^{\mathrm{eq}} / M$ will be zero and the intercept will be finite. Then, using aquatic density $1.0 \mathrm{~g} / \mathrm{ml}$ for the solvent and $1.8 \mathrm{~g} / \mathrm{ml}$ for DNA [66], a typical dissipation factor of $\mathrm{ca} .10^{-4}$ for the seventh harmonic $(35 \mathrm{MHz})$ and the value $w=42.0$ that we determined previously, we find an intercept $\approx 0.00029 \times\left(10^{-6} / \mathrm{Hz}\right)$. This is approximately 40 times lower than the experimental result of $0.0112 \times\left(10^{-6} / \mathrm{Hz}\right)$ for dsDNA at $35 \mathrm{MHz}$, determined in Refs. [23,24], implying that the main contribution comes from the first term in the rhs of Eq. (B10), which is only possible if the equivalent hydrodynamic radius varies linearly with molecular weight or

$$
\lim _{M \rightarrow 0} \frac{r_{h}^{\text {eq }}}{M}=K_{r} \quad \text { (constant). }
$$

This is used in the main text to estimate the proportionality coefficient by which $r_{h}^{\mathrm{eq}}$ depends on $M$.

Generally, the way the equivalent hydrodynamic radius varies with molecular weight is not known in advance, but can be found by fitting some appropriate expression to experimental data. Given enough data, fitting more than one parameter is possible and both radiusgoverning parameters and analyte molecule density can be determined. It is reminded that the equivalent radius does not indicate the actual size of analyte molecules. By its definition, $r_{h}^{\text {eq }}$ is a measure of how much the particle's velocity deviates from that of the surrounding fluid. Although not accounted for explicitly in our model, the stiffness of the analyte molecule-surface link and the analyte molecule's internal degrees of freedom should affect the extent to which particles and fluid coordinate as reflected in the fitted values of the equivalent radius. Therefore, we expect that the intercept is closely related with the nature of the linker as well as the analyte molecule's flexibility. Importantly, both slope and intercept are independent of surface coverage.

[1] G. Sauerbrey, Verwendung von schwingquarzen zur wägung dünner schichten und zur mikrowägung, Zeitschrift Phys. 155, 206 (1959).

[2] J. Krim, Friction and energy dissipation mechanisms in adsorbed molecules and molecularly thin films, Adv. Phys. 61, 155 (2012).
[3] L. Bruschi and G. Mistura, Measurement of the friction of thin films by means of a quartz microbalance in the presence of a finite vapor pressure, Phys. Rev. B 63, 235411 (2001).

[4] K. K. Kanazawa and J. G. Gordon, The oscillation frequency of a quartz resonator in contact with a liquid, Anal. Chim. Acta 175, 99 (1985).

[5] F. F. Ouali, N. Doy, G. McHale, C. Hardacre, R. Ge, R. W. K. Allen, J. M. MacInnes, and M. I. Newton, Determination of the physical properties of room temperature ionic liquids using a Love wave device, Anal. Chem. 83, 6717 (2011).

[6] M. V. Voinova, M. Rodahl, M. Jonson, and B. Kasemo, Viscoelastic acoustic response of layered polymer films at fluid-solid interfaces: Continuum mechanics approach, Phys. Scr. 59, 391 (1999).

[7] D. Johannsmann, K. Mathauer, G. Wegner, and W. Knoll, Viscoelastic properties of thin films probed with a quartz crystal resonator, Phys. Rev. B 46, 7808 (1992).

[8] D. Johannsmann, Viscoelastic analysis of organic thin films on quartz resonators, Macromol. Chem. Phys. 200, 501 (1999).

[9] J. de-Carvalho, R. M. M. Rodrigues, B. Tomé, S. F. Henriques, N. P. Mira, I. Sá-Correia, and G. N. M. Ferreira, Conformational and mechanical changes of DNA upon transcription factor binding detected by a QCM and transmission line model, Analyst 139, 1847 (2014).

[10] H. L. Bandey, S. J. Martin, R. W. Cernosek, and A. R. Hillman, Modeling the responses of thickness-shear mode resonators under various loading conditions, Anal. Chem. 71, 2205 (1999).

[11] R. Lucklum, C. Behling, R. W. Cernosek, and S. J. Martin, Determination of complex shear modulus with thickness shear mode resonators, J. Phys. D-Appl. Phys. 30, 346 (1997).

[12] N. Liu, J. S. Yang, and W. Q. Chen, Effects of a mass layer with gradually varying thickness on a quartz crystal microbalance, IEEE Sens. J. 11, 1635 (2011).

[13] G. Fischerauer and F. L. Dickert, A simple model for the effect of nonuniform mass loading on the response of gravimetric chemical sensors, Sens. Actuators B Chem. 229, 618 (2016).

[14] L. Daikhin, E. Gileadi, G. Katz, V. Tsionsky, M. Urbakh, and D. Zagidulin, Influence of roughness on the admittance of the quartz crystal microbalance immersed in liquids, Anal. Chem. 74, 554 (2002).

[15] G. L. Dybwad, A sensitive new method for the determination of adhesive bonding between a particle and a substrate, J. Appl. Phys. 58, 2789 (1985).

[16] G. L. Dybwad, Sensitive New Method for the Determination of Adhesion Force Between a Particle and a Substrate, edited by K. L. Mittal, Particles on Surfaces: Detection, Adhesion and Removal (Plenum Press, New York, 1988), pp. 237-243.

[17] D. Johannsmann, I. Reviakine, and R. P. Richter, Dissipation in films of adsorbed nanospheres studied by quartz crystal microbalance (QCM), Anal. Chem. 81, 8167 (2009).

[18] E. Tellechea, D. Johannsmann, N. F. Steinmetz, R. P. Richter, and I. Reviakine, Model-independent analysis of QCM data on colloidal particle adsorption, Langmuir 25, 5177 (2009). 
[19] D. Johannsmann and G. Brenner, Frequency shifts of a quartz crystal microbalance calculated with the frequencydomain Lattice-Boltzmann method: application to coupled liquid mass, Anal. Chem. 87, 7476 (2015).

[20] J. J. J. Gillissen, S. R. Tabaei, J. A. Jackman, and N. J. Cho, A model derived from hydrodynamic simulations for extracting the size of spherical particles from the quartz crystal microbalance, Analyst 142, 3370 (2017).

[21] J. J. J. Gillissen, J. A. Jackman, S. R. Tabaei, and N. J. Cho, A numerical study on the effect of particle surface coverage on the quartz crystal microbalance response, Anal. Chem. 90, 2238 (2018).

[22] A. Tsortos, G. Papadakis, K. Mitsakakis, K. A. Melzak, and E. Gizeli, Quantitative determination of size and shape of surface-bound DNA using an acoustic wave sensor, Biophys. J. 94, 2706 (2008).

[23] A. Tsortos, G. Papadakis, and E. Gizeli, Shear acoustic wave biosensor for detecting DNA intrinsic viscosity and conformation: A study with QCM-D, Biosens. Bioelectron. 24, 836 (2008).

[24] A. Tsortos, G. Papadakis, and E. Gizeli, On the hydrodynamic nature of DNA acoustic sensing, Anal. Chem. 88, 6472 (2016).

[25] D. Milioni, A. Tsortos, M. Velez, and E. Gizeli, Extracting the shape and size of biomolecules attached to a surface as suspended discrete nanoparticles, Anal. Chem. 89, 4198 (2017).

[26] G. Papadakis, A. Tsortos, F. Bender, E. E. Ferapontova, and E. Gizeli, Direct detection of DNA conformation in hybridization processes, Anal. Chem. 84, 1854 (2012).

[27] P. Mateos-Gil, A. Tsortos, M. Velez, and E. Gizeli, Monitoring structural changes in intrinsically disordered proteins using QCM-D: application to the bacterial cell division protein ZipA, Chem. Commun. 52, 6541 (2016).

[28] A. Tsortos, G. Papadakis, and E. Gizeli, The intrinsic viscosity of linear DNA, Biopolymers 95, 824 (2011).

[29] M. L. Mansfield, A. Tsortos, and J. F. Douglas, Persistent draining crossover in DNA and other semi-flexible polymers: Evidence from hydrodynamic models and extensive measurements on DNA, J. Chem. Phys. 143, 124903 (2015).

[30] S. E. Harding, The intrinsic viscosity of biological macromolecules. Progress in measurement, interpretation and application to structure in dilute solution, Prog. Biophys. Mol. Biol. 68, 207 (1997).

[31] J. Garcia de la Torre and V. A. Bloomfield, Hydrodynamic properties of complex, rigid, biological macromolecules: theory and applications. Q. Rev. Biophys. 14, 81 (1981).

[32] C. Tanford, Physical Chemistry of Macromolecules (Wiley, New York, 1961).

[33] C. R. Cantor and P. R. Schimmel, Biophysical Chemistry, Part II (Freeman, San Francisco, 1980).

[34] J. Garcia de la Torre and V. A. Bloomfield, Hydrodynamic properties of macromolecular complexes IV. Intrinsic viscosity theory, with applications to once-broken rods and multisubunit proteins, Biopolymers 17, 1605 (1978).

[35] A. Ortega, D. Amoros, and J. Garcia de la Torre, Prediction of hydrodynamic and other solution properties of rigid proteins from atomic and residue-level models, Biophys. J. 101, 892 (2011).
[36] D. Amoros, A. Ortega, and J. Garcia de la Torre, Prediction of hydrodynamic and other solution properties of partially disordered proteins with a simple, coarse-grained model, J. Chem. Theory Comput. 9, 1678 (2013).

[37] W. Tang, D. Z. Wang, Y. Xu, N. Li, and F. Liu, A self-assembled DNA nanostructure-amplified quartz crystal microbalance with dissipation biosensing platform for nucleic acids, Chem. Commun. 48, 6678 (2012).

[38] R. H. Walters, K. H. Jacobson, J. A. Pedersen, and R. M. Murphy, Elongation kinetics of polyglutamine peptide fibrils: a quartz crystal microbalance with dissipation study, J. Mol. Biol. 421, 329 (2012).

[39] D. Zhu, H. Pei, G. Yao, L. Wang, S. Su, J. Chao, L. Wang, A. Aldalbahi, S. Song, J. Shi, J. Hu, C. Fan, and X. Zuo, A surface-confined proton-driven DNA pump using a dynamic 3D DNA scaffold, Adv. Mater. 28, 6860 (2016).

[40] I. E. Ivanov, A. E. Morrison, J. E. Cobb, C. A. Fahey, and T. A. Camesano, Creating antibacterial surfaces with the peptide chrysophsin, ACS Appl. Mater. Interfaces 4, 5891 (2012).

[41] A. Osypova, D. Thakar, J. Dejeu, H. Bonnet, A. Van der Heyden, G. V. Dubacheva, R. P. Richter, E. Defrancq, N. Spinelli, L. Coche-Guérente, and P. Labbé, Sensor based on aptamer folding to detect low-molecular weight analytes, Anal. Chem. 87, 7566 (2015).

[42] N. Giamblanco, S. Petralia, S. Conoci, C. Messineo, and G. Marletta, Ionic strength-controlled hybridization and stability of hybrids of KRAS DNA single-nucleotides: A surface plasmon resonance study, Colloids Surf. B Biointerfaces 158, 41 (2017).

[43] I. Reviakine, D. Johannsmann, and R. P. Richter, Hearing what you cannot see and visualizing what you hear: interpreting quartz crystal microbalance data from solvated interfaces, Anal. Chem. 83, 8838 (2011).

[44] M. Snejdarkova, A. Poturnayová, P. Rybár, P. Lhoták, M. Himl, K. Flídrová, and T. Hianik, High sensitive calixarenebased sensor for detection of dopamine by electrochemical and acoustic method, Bioelectrochemistry 80, 55 (2010).

[45] J. S. Ellis and M. Thompson, Acoustic physics of surfaceattached biochemical species, HFSP J. 2, 171 (2008).

[46] X. Du, J. Fang, and D. M. Zhu, Modified Sauerbrey equation: a facile method to quantitatively probe the conformation of isolated molecules at solid-liquid interfaces, Analyst 143, 3209 (2018).

[47] A. Tsortos, A. Grammoustianou, R. Lymbouridou, G. Papadakis, and E. Gizeli, The detection of multiple DNA targets with a single probe using a conformation-sensitive acoustic sensor, Chem. Commun. 51, 11504 (2015).

[48] G. Papadakis, A. Tsortos, A. Kordas, I. Tiniakou, E. Morou, J. Vontas, D. Kardassis, and E. Gizeli, Acoustic detection of DNA conformation in genetic assays combined with PCR, Sci. Rep. 3, 2033 (2013).

[49] G. Papadakis, P. Palladino, D. Chronaki, A. Tsortos, and E. Gizeli, Sample-to-answer acoustic detection of DNA in complex samples, Chem. Commun. 53, 8058 (2017).

[50] G. Papadakis and E. Gizeli, Screening for mutations in BRCA1 and BRCA2 genes by measuring the acoustic ratio with QCM, Anal. Methods 6, 363 (2014).

[51] G. Papadakis, A. Tsortos, K. Mitsakakis, and E. Gizeli, Characterization of DNA-Hv1 histone interactions; 
discrimination of DNA size and shape, FEBS Lett. 584, 935 (2010).

[52] G. Papadakis, A. Tsortos, and E. Gizeli, Triple-helix DNA structural studies using a Love wave acoustic biosensor, Biosens. Bioelectron. 25, 702 (2009).

[53] G. Papadakis, A. Tsortos, and E. Gizeli, Acoustic characterization of nanoswitch structures: application to the DNA Holliday junction, Nano Lett. 10, 5093 (2010).

[54] V. M. Mecea, Loaded vibrating quartz sensors, Sens. Actuators A Phys. 40, 1 (1994).

[55] M. Hopfner, U. Rothe, and G. Bendas, Biosensor-based evaluation of liposomal behavior in the target binding process, J. Liposome Res. 18, 71 (2008).

[56] B. Pignataro, C. Steinem, H. J. Galla, H. Fuchs, and A. Janshoff, Specific adhesion of vesicles monitored by scanning force microscopy and quartz crystal microbalance, Biophys. J. 78, 487 (2000).

[57] F. N. Dultsev, V. P. Ostanin, and D. Klenerman, 'Hearing' bond breakage; measurement of bond rupture forces using a quartz crystal microbalance, Langmuir 16, 5036 (2000).

[58] A. M. Esfahani, W. Zhao, J. Y. Chen, C. Huang, N. Xi, J. Xi, and R. Yang, On the measurement of energy dissipation of adhered cells with the quartz microbalance with dissipation monitoring, Anal. Chem. 90, 10340 (2018).

[59] W. E. Langlois and M. O. Deville, Slow Viscous Flow (Springer, Heidelberg, 2014), 2nd ed.

[60] H. Lamb, Hydrodynamics (Dover, Mineola, NY, 1945).

[61] S. Kim and S. J. Karrila, Microhydrodynamics (Dover, Mineola, NY, 2005).

[62] C. Kittel, W. D. Knigth, M. A. Ruderman, A. C. Helmholz, and B. J. Moyer, Mechanics - Berkeley Physics Course (McGraw-Hill, New York, 1973), 2nd ed., Vol. I.

[63] W. A. Hendrickson, A. Pahler, J. L. Smith, Y. Satow, E. A. Merritt, and R. P. Phizackerley, Crystal structure of core streptavidin determined from multiwavelength anomalous diffraction of synchrotron radiation, Proc. Natl. Acad. Sci. U.S.A. 86, 2190 (1989).

[64] J. A. Nelder and R. Mead, A simplex method for function minimization, Comput. J. 7, 308 (1965).

[65] D. G. Papageorgiou, I. N. Demetropoulos, and I. E. Lagaris, MERLIN-3.1.1. A new version of the Merlin optimization environment, Comput. Phys. Commun. 159, 70 (2004).

[66] J. E. Hearst, The specific volume of various cationic forms of deoxyribonucleic acid, J. Mol. Biol. 4, 415 (1962). 